TRANSACTIONS OF THE

AMERICAN MATHEMATICAL SOCIETY

Volume 348, Number 12, December 1996, Pages 5031-5063

S $0002-9947(96) 01578-4$

\title{
SPATIAL CHAOTIC STRUCTURE OF ATTRACTORS OF REACTION-DIFFUSION SYSTEMS
}

\author{
V. AFRAIMOVICH, A. BABIN, AND S.-N. CHOW
}

\begin{abstract}
The dynamics described by a system of reaction-diffusion equations with a nonlinear potential exhibits complicated spatial patterns. These patterns emerge from preservation of homotopy classes of solutions with bounded energies. Chaotically arranged stable patterns exist because of realizability of all elements of a fundamental homotopy group of a fixed degree. This group corresponds to level sets of the potential. The estimates of homotopy complexity of attractors are obtained in terms of geometric characteristics of the potential and other data of the problem.
\end{abstract}

\section{INTRODUCTION}

We describe here a mechanism which causes complicated spatial behavior of solutions of PDE. This mechanism is robust, and conditions under which it works are very simple to verify. It may explain chaotic spatial patterns arising in many branches of mechanics, physics, material science, biology, etc. The main cause of such chaotic patterns due to our approach is the persistence of certain homotopy classes under the dynamics of the system. They are discrete-valued conserved quantities. These homotopy classes are determined in terms of nonlinearities involved in considered equations. The number of different conserved classes exponentially depends on parameters of the systems. Symbolic dynamics describing chaos is determined by homotopy groups. The essential feature of our approach is that it is based not on local properties of solutions of the equations (such as, for example, homoclinicity) but rather on studying global quantities, such as the energy of the system. Note that we describe in the considered situations regular middle-scale patterns which are irregularly (chaotically) distributed on a larger scale and not a high-frequency chaotic behavior which may happen in other problems.

We consider semilinear parabolic systems of the form

$$
\partial_{t} u=a \partial_{x}^{2} u-F^{\prime}(u) .
$$

Here $F$ is a function of class $C^{1}\left(\mathbb{R}^{d}\right), d \geq 2, F^{\prime}$ is its gradient, $u=\left(u_{1}, \ldots, u_{d}\right)$, $x \in[0, L] \subset \mathbb{R}, F(u) \geq 0 \forall u \in \mathbb{R}^{d}$. We impose Dirichlet boundary conditions

$$
\left.u\right|_{x=0}=\left.u\right|_{x=L}=b, \quad b \in \mathbb{R}^{d},
$$

Received by the editors July 18, 1994 and, in revised form, June 22, 1995.

1991 Mathematics Subject Classification. Primary 35K57; Secondary 34C35.

Key words and phrases. Reaction-diffusion system; potential; homotopy complexity; symbolic dynamics.

The first and third authors were partially supported by ARO DAAH04-93G-0199.

Research was partially supported by NIST Grant 60NANB2D1276.

(C)1996 American Mathematical Society 


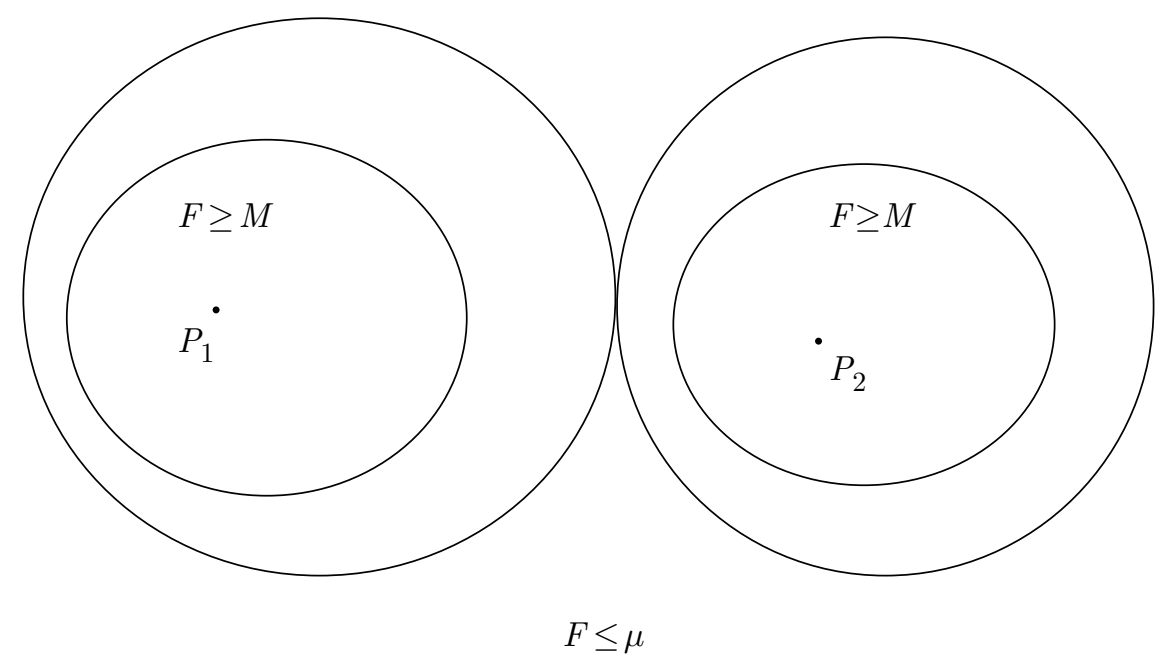

FigURE 1

or periodic boundary conditions

$$
\left.u\right|_{x=0}=\left.u\right|_{x=L},\left.\quad \partial_{x} u\right|_{x=0}=\left.\partial_{x} u\right|_{x=L} .
$$

Complicated spatial patterns persist under dynamics generated by this equation if variation of the potential $F$ is large enough (for more precise conditions on $F$ see below). The dynamics generated by (0.1) preserves homotopy classes determined by the potential $F$ and initial data $u_{0}$ if the energy of initial data is not very large. Nontrivial homotopy classes correspond to complex spatial patterns of solutions, these patterns change with time but their homotopy type and, therefore, complexity is preserved. The homotopy type of these patterns is preserved also under continuous perturbations of initial data (with a bounded energy). The cause which generates persistent complicated spatial patterns is the structure of level sets and is a strong enough variation of the function $F$. We discuss it in the introduction in the case $d=2$. To provide complicated solutions, the function $F$ suffices to have at least two relatively sharp local maxima, the value of which should be large enough with respect to values of $F$ in a low-energy domain $\mathcal{D}_{\mu}$, which surround them. To be more specific, assume that we have two points $P_{1}, P_{2} \in \mathbb{R}^{2}\left(P_{i}=\left(u_{i 1}, u_{i 2}\right)\right)$, $\left|P_{1}-P_{2}\right|=2 R$, such that the potential $F$ is large in $r$-neighborhood of these points $(r<R)$,

$$
F(u) \geq M \quad \text { when }\left|u-P_{1}\right| \leq r \text { or }\left|u-P_{2}\right| \leq r .
$$

The potential has to be small outside larger neighborhoods of these points,

$$
F(u) \leq \mu \quad \text { when }\left|u-P_{1}\right| \geq R,\left|u-P_{2}\right| \geq R
$$

(see Figure 1).

We denote the union of discs of radius $r$ surrounding $P_{1}$ and $P_{2}$ by $\Omega(P, r)$, and its complement in $\mathbb{R}^{2}$ by $\Omega(P, r), \Omega^{\prime}(P, r)=\mathbb{R}^{2} \backslash \Omega(P, r)=\left\{u:\left|u-P_{i}\right|>r, i=\right.$ $1,2\}$. The topology of $\Omega^{\prime}$ is described by its fundamental group $\pi_{1}\left(\Omega^{\prime}(P, r)\right)$. This group corresponds to classes of continuous mappings of a circle $S^{1}$ into $\Omega^{\prime}$, two homotopy equivalent mappings $u: S^{1} \rightarrow \Omega^{\prime}$ correspond to the same class $g(u)$. 


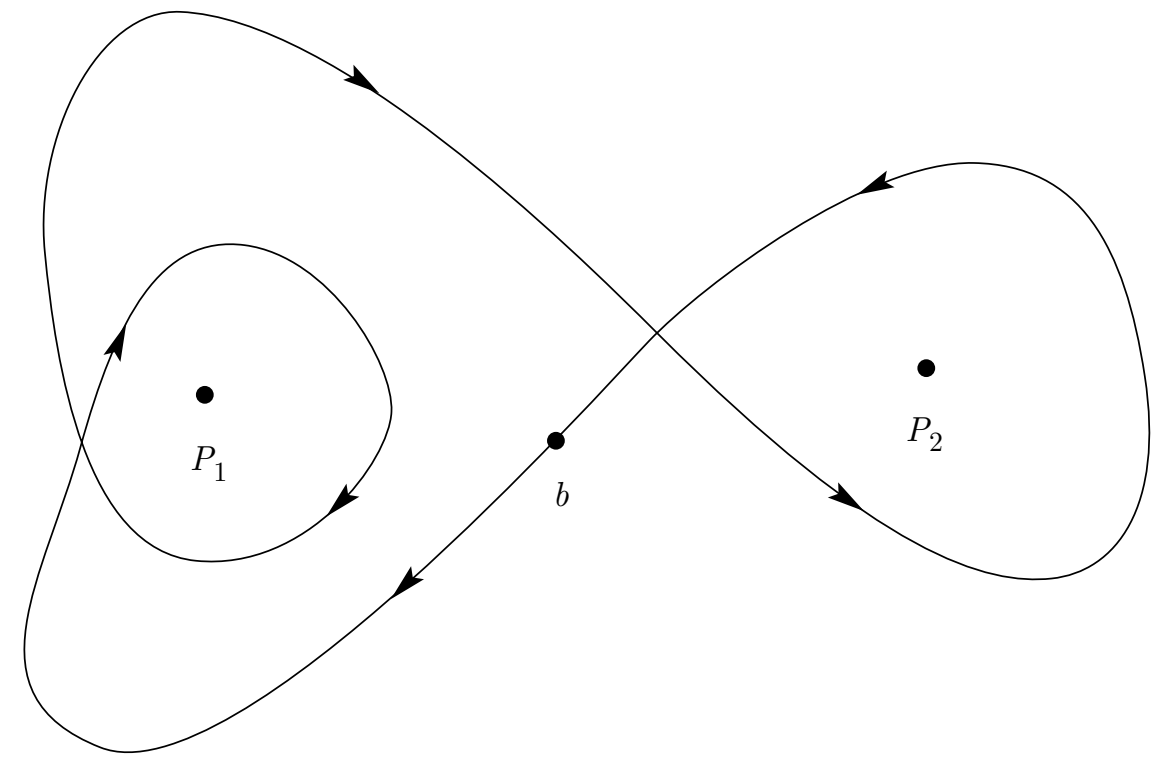

Figure 2

We shall consider a circle $S^{1}$ as a segment $[0, L]$ with endpoints glued together, so $u(0)=u(L)$. An example of the class is represented in Figure 2 .

This class represents two clockwise turns around $P_{1}$ and one counterclockwise turn around $P_{2}$; it can be written as an element $g \in \pi_{1}\left(\Omega^{\prime}(P, r), *\right)$ in the form

$$
g=g_{1}^{-2} g_{2}
$$

where generators $g_{1}$ and $g_{2}$ correspond to counterclockwise turns around $P_{1}$ and $P_{2}$ respectively. For brevity, we shall consider mappings with a base point $* \in S^{1}$, $*=0=L$, and another base point $b \in \Omega^{\prime}(P, r)$. (This case corresponds to Dirichlet boundary conditions. The case of periodic boundary conditions is similar but a little more complicated.) In this case the homotopy group is as usual denoted by $\pi_{1}\left(\Omega^{\prime}, *\right)$. Obviously, $\pi_{1}\left(\Omega^{\prime}(P, r), *\right)$ does not depend on $r, 0<r<R$. An arbitrary finite sequence of non-zero integers $k_{1}, \ldots, k_{n}$ determines an element

$$
g=g_{i_{1}}^{k_{1}} g_{i_{2}}^{k_{2}} \ldots g_{i_{n}}^{k_{n}}
$$

$i_{j+1} \neq i_{j}$. The sum $\left|k_{1}\right|+\cdots+\left|k_{n}\right|=\operatorname{deg} g$ is called the degree of a monomial $g$. Obviously, $g$ also can be represented as a product of $\operatorname{deg} g$ elements $g_{1}, g_{2}, g_{1}^{-1}, g_{2}^{-1}$, so the elements of the group $G=\pi_{1}\left(\Omega^{\prime}(P, r)\right.$,*) can be represented as finite words which consist of these four symbols and are subjected to a restriction: it should not include combinations $g_{1} g_{1}^{-1}, g_{1}^{-1} g_{1}, g_{2} g_{2}^{-1}, g_{2}^{-1} g_{2}$. If $\operatorname{deg} g$ is fixed, there is no less than $3^{\operatorname{deg} g}$ of such words of length deg $g$. (More precisely, $4 \cdot 3^{\operatorname{deg} g-1}$ in the case of Dirichlet boundary conditions.)

The structure of the word $g$ contains information on the spatial structure corresponding to the function $u \in g$ (recall, that $g$ is an equivalence class, therefore we write inclusion). For example, if points $P_{1}$ and $P_{2}$ have coordinates $\left(-R_{1}, 0\right)$ and $\left(R_{1}, 0\right)$ respectively and $b=(0,0)$, then the function $u_{1}(x)$ which corresponds to the curve $u(x)$ described in Figure 2, has the graph of the form described in Figure 3. 


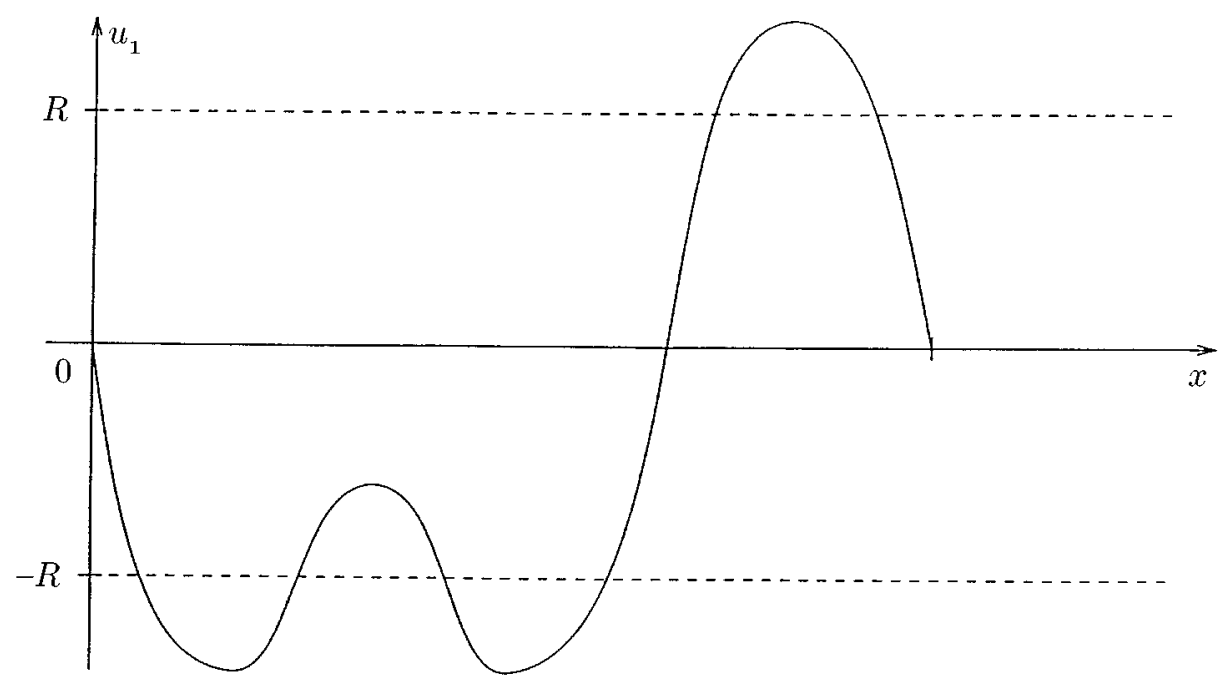

FiguRE 3

Sharp negative minima correspond to $g_{1}$ or $g_{1}^{-1}$, and sharp positive maxima to $g_{2}$ or $g_{2}^{-1}$. Analogously a graph of the form shown in Figure 4 corresponds to an element of form (0.6) $g=g_{2}^{k_{1}} g_{1}^{k_{2}} g_{2}^{k_{3}} g_{1}^{k_{4}}$ with $i_{1}=2,\left|k_{1}\right|=3, i_{2}=1,\left|k_{2}\right|=2, i_{3}=2$, $\left|k_{3}\right|=1, i_{4}=1,\left|k_{4}\right|=4$. (To see the difference between positive and negative $k_{j}$, we have to consider $u_{2}(x)$ as well.) One can see that neighboring terms $g_{1}^{ \pm 1} g_{2}^{ \pm 1}$ or $g_{2}^{ \pm 1} g_{1}^{ \pm 1}$ create transitions of amplitude of order $R$, and $g_{1}$ or $g_{2}$ itself are of order $r$ (this can be easily justified if $M \gg \mu, R \gg r$ ). Hence, we have 4 transitions of order $R$ and 10 oscillations of order $r$ in this picture. So, if we have an element

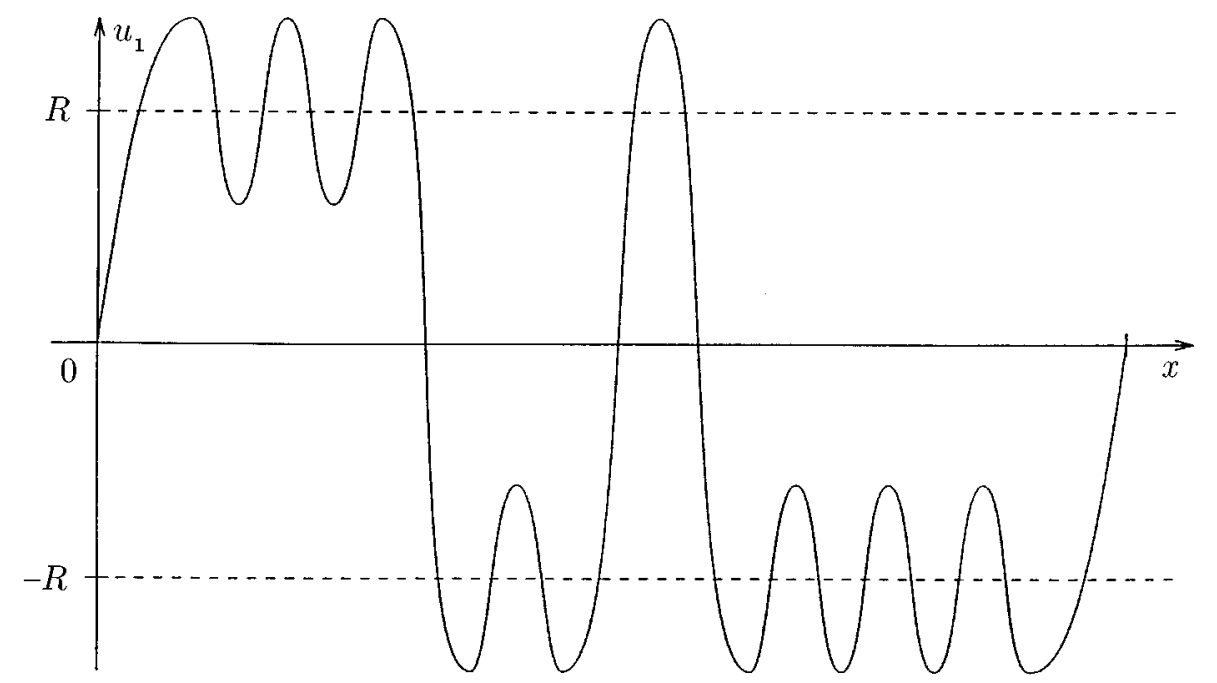

FiguRE 4 
$g \in G$, its representative $u \in g$ has a homotopy stable spatial structure described by $u(x)$, which cannot be simpler than the structure of the element $g$ itself.

Therefore, if we have at a fixed time $t$ solution $u(x, t)$, such that $u(\cdot, t) \in g$ where $g \in G$ has a complex structure, then $u(x, t)$ has a complex structure as a function of $x$. If an attractor $\mathcal{A}$ includes $N$ elements (which belong to different homotopy types), we call $\ln N$ homotopy complexity of the attractor.

To describe behavior of the system (0.1), it is very useful to consider the functional

$$
\mathcal{P}(u)=\int_{0}^{L}\left(\frac{a}{2}\left|\partial_{x} u\right|^{2}+F(u)\right) d x .
$$

(It can be considered as the energy of the function $u$.) If $u(x, t)$ is a solution of (0.1), (0.2), then $\mathcal{P}(u(t))$ is a monotone non-increasing function of $t$.

It is well known (see $[\mathrm{BV}])$, that the system $(0.1),(0.2)$ generates dynamics in Sobolev's space $H_{1}([0, L])$ and has a global attractor $\mathcal{A}$. For any initial function $u(0) \in H_{1}([0, L])$ the solution $u(t)$ after some time (which depends only on the norm of $u(0)$ in Sobolev space $\left.H_{1}([0, L])\right)$ gets arbitrarily close to the global attractor $\mathcal{A}$. Therefore, the solutions of (0.1) have complicated spatial structure for large $t$ if the attractor consists of complex functions $v(x)$.

The sets $E_{\beta}=\left\{u \in H_{1}[0, L]: \mathcal{P}(u) \leq \beta\right\}$ are invariant with respect to these dynamics, these sets have attractors $\mathcal{A}(\beta)$. The main observation which we use in this paper is that dynamics generated by (0.1) on $E_{\beta}$ if $\beta$ is not too large, has a number of conserved quantities, namely these quantities are homotopy classes $g(u(t))=g \in \pi_{1}\left(\mathbb{R}^{2} \backslash\left(P_{1} \cup P_{2}\right)\right)$ of solutions $u(t)$ of $(0.1)$. Sufficient condition on $\beta$ under which $g(u)$ is well-defined is

$$
\beta<r \sqrt{8 M a}
$$

(see Theorems 2.1, 2.6, 4.1). The sets $\left\{u \in E_{\beta}, g(u)=g\right\}=Y(g, \beta)$ are nonempty if the following sufficient condition (we assume here that $\left|P_{1}-P_{2}\right|=2 R$, $\left.b=\left(P_{1}+P_{2}\right) / 2\right)$ is satisfied:

$$
\operatorname{deg} g \leq Q^{1 / 2}, \quad Q=\frac{r \sqrt{8 M} L}{\sqrt{a}(2 \pi R)^{2}}-\frac{L^{2} \mu}{a(2 \pi R)^{2}} .
$$

(There exist at least $3^{\operatorname{deg} g}$ different elements $g \in G$ with given deg $g$.) In particular, under these conditions for every element $g \in G$, deg $g \leq d_{0}$, there exists a stable component $\mathcal{A}(\beta, g) \subset \mathcal{A}_{\beta}$ of the attractor $\mathcal{A}_{\beta}$, such that all elements of $\mathcal{A}(\beta, g)$ consist of $v \in g$. Therefore, all possible functions of the form shown in Figure 4 are represented as limit (as $t \rightarrow+\infty$ ) spatial patterns of solutions of (0.1), all sequences of $\left(k_{1}, \ldots, k_{n}\right)$, describing the number of sharp maxima are realizable, provided $\left|k_{1}\right|+\cdots+\left|k_{n}\right| \leq d_{0}$.

For example, consider the most simple situation of a singular potential. Let

$$
F(u) \geq q\left(\left|u-P_{1}\right|^{-3}+\left|u-P_{2}\right|^{-3}\right)
$$

when $u \in \Omega(P, r)$, with $r>0, q>0$ and let $F(u) \leq \mu$ when $u \in \Omega^{\prime \prime}(P, r)=\{|u| \leq$ $3 R\} \cap\left\{\Omega^{\prime}(P, r)\right\}$. Then we obtain $\sqrt{M} r \rightarrow \infty$ as $r \rightarrow 0$. We can take energy level $\beta$ arbitrarily large and there exist functions $u \in g \cap E_{\beta}$ with arbitrarily large deg $g$. Therefore, we obtain arbitrarily complex spatial patterns described by arbitrary $g$. 
Hence, in the situation of a strongly singular potential satisfying (0.10), arbitrary configurations of the type described in Figure 4 are realizable as spatial forms of solutions $u(x, t)$ on the fixed interval $[0, L]$.

Note that the case of nonsingular potential is more complicated (and may be more interesting). In this case for solutions with large initial energy $\mathcal{P}(u(0))$ the homotopy class may not be defined. But $\mathcal{P}(u(t))$ is a strictly decreasing function of time, and after some time the condition $\mathcal{P}(u(t))<\beta$ may be fulfilled. Such a behavior can be interpreted as the emerging of a (complicated) spatial middlescale pattern of originally chaotic (on small scales) patterns without distinguishable middle-scale elements corresponding to homotopy groups. This recalls a process of crystallization under decreasing temperature.

We will discuss briefly now the formulae which give restrictions on deg $g$ of homotopy classes to which solutions belong. These inequalities give estimates of complexity of solutions in very simple terms.

The right-hand side of (0.9) is maximal if we take $L=r \sqrt{2 a M} / \mu$ and we obtain

$$
Q=\frac{r^{2} M}{\pi^{2} R^{2} \mu} .
$$

The important feature of the situation when complex patterns persist is that there are at least two different levels $M$ (high) and $\mu$ (low) of potential energy $F$ and two different characteristic lengths $r$ and $R$ in the $\left(u_{1}, u_{2}\right)$ plane. The length $r$ is proportional to the minimal width of high-energy level core. The length $R$ is proportional to the length of a cycle turning around a location of high potential energy but remaining in the low energy zone. The characteristic quantities are $\sqrt[4]{M r^{2}}=\sigma_{\text {high }}$ and $\sqrt[4]{\mu R^{2}}=\sigma_{\text {low }}$; they are of dimensionality of the $u$. The chaotic pattern persists if $\sigma_{\text {high }} \gg \sigma_{\text {low }}$ and the complexity describing the chaos is proportional to the ratio $\frac{\sigma_{\text {high }}}{\sigma_{\text {low }}}, \operatorname{deg} g \leq C \frac{\sigma_{\text {high }}}{\sigma_{\text {low }}}$ is the limitation on the degree of the homotopy element $g$ (roughly speaking, on the number of crests in the graphs of $u_{1}$ and $u_{2}$ ).

In the situation when $L$ in $(0.2 \mathrm{a})$ or $(0.2 \mathrm{~b})$ is not prescribed, the quantities $\sigma_{\text {high }}$ and $\sigma_{\text {low }}$ which characterize the potential $F$ play determining roles. If $L$ is fixed, a new scaling parameter appears and one more quantity $\sigma_{L}=R \sqrt[4]{a} / \sqrt{L}$ of dimensionality of $u$ is of importance. One can easily see that the quantity $Q$ described by (0.9) is proportional to

$$
Q_{0}=\left(\frac{\sigma_{\text {high }}}{\sigma_{L}}\right)^{2}-\left(\frac{\sigma_{\text {low }}}{\sigma_{L}}\right)^{4}
$$

and complexity is proportional to

$$
\sqrt{\left(\frac{\sigma_{\text {high }}}{\sigma_{L}}\right)^{2}-\left(\frac{\sigma_{\text {low }}}{\sigma_{L}}\right)^{4}} .
$$

Now we make remarks on equilibriums of (0.1). The steady states of (0.1) are solutions of system of ODE

$$
a \partial_{x}^{2} u-F^{\prime}(u)=0 .
$$

To use notations closer to standard for ODE, we put $x=\tau, \partial_{x} u=\dot{u}=P$, $-F(u)=U(u)$ and $H(u, p)=U(u)+\frac{a}{2} p^{2}$. Then (0.11) can be written in the form of Hamiltonian systems $\dot{u}=H_{p}, \dot{p}=-H_{p}$. Our results imply existence of a set of solutions of this system with positive entropy. Existence of many solutions of 
problems of type (0.11) was proved in many papers, see $[\mathrm{G}],[\mathrm{B}],[\mathrm{R}],[\mathrm{ACZ}]$. Our approach is different in the respect that we connect the number of solutions with values of $\mathcal{P}$ and not $H$, obtaining exponentially many different stable solutions, and we use the theory of dynamical systems in infinite dimensional spaces.

Another method was used in $[\mathrm{N}]$ where existence of many (but not exponentially many) solutions was proved for reaction-diffusion systems with large ratio of diffusion rates for different components.

Complex structure of the spatial domain can also cause existence of many stable solutions (see $[\mathrm{JM}],[\mathrm{H}]$ where harmonic mappings of different topological types are constructed). In this approach, contrary to ours, topology of solutions is prescribed from the very beginning. In a way, our approach is closer to $[\mathrm{BBH}]$ where topology arises in the dynamics when singularities generate non-trivial topology of the spatial domain. Our approach is applicable to more general situations and gives, unlike in papers cited, exponentially many different types of stable solutions. We took the idea to connect complicated spatial behavior of solutions with symbolic dynamics from $[\mathrm{AC}]$. In that work solutions on lattices were considered. Since we have solutions on a connected set in the present work, we use homotopy-class valued symbolic dynamics here. One has to note that the structure of nonlinearities and boundary conditions play very important role in our approach. Without this kind of assumption one cannot hope to obtain non-homogeneous stable solutions (see $[\mathrm{KW}])$.

Generalizations of results of the paper are possible in many directions; we mention some of them. The first concerns the case of $x$-dependent potential $F(x, u)$, $x \in S^{1}$. Under appropriate conditions the homotopy classes of sections of fiber bundles over $S^{1}$ are preserved.

Another direction is the case of many spatial variables, $x \in \Omega \subset \mathbb{R}^{n}, n>1$. In this case dynamics described by quasilinear systems preserves homotopy classes of mappings $u: \Omega \rightarrow \mathbb{R}^{d}$ (with appropriate boundary conditions and conditions on $F$ and main part).

These and other generalizations will be published elsewhere.

\section{A Semigroup Generated by a Parabolic Equation}

We consider a semilinear parabolic system of equations

$$
\partial_{t} u=a \partial_{x}^{2} u-F^{\prime}(u, x)
$$

where $a>0,0<x<L, u=\left(u_{1}, u_{2}, \ldots, u_{d}\right), d \geq 2, F: \mathbb{R}^{d} \times[0, L] \rightarrow \mathbb{R}$ is a $C^{1}$ function (a potential), $F^{\prime}=\left(\partial F / \partial u_{1}, \ldots, \partial F / \partial u_{d}\right)=\nabla F$ is a Lipschitz function of $u, \partial_{x} u=\partial u / \partial x, \partial_{t} u=\partial u / \partial t$. We impose one of two boundary conditions: either Dirichlet,

$$
\left.u\right|_{x=0}=b_{1},\left.\quad u\right|_{x=L}=b_{2}
$$

(for simplicity we shall take $b_{1}=b_{2}=b$ - the case $b_{1} \neq b_{2}$ makes no difference in this section but may cause more lengthy discussion later); or the periodic (in this case $F$ is assumed to be periodic in $x$ as well),

$$
\left.u\right|_{x=0}=\left.u\right|_{x=L}=0,\left.\quad \partial_{x} u\right|_{x=0}=\left.\partial_{x} u\right|_{x=L} .
$$


(We shall refer to these conditions as (1.2) if both are treated in the same way.) We assume that the potential $F$ satisfies the condition

$$
\begin{gathered}
F(u, x) \geq 0 \quad \forall u, x ; \\
\left|F^{\prime}(u, x)-F^{\prime}(v, x)\right| \leq C(|u|,|v|)|u-v|
\end{gathered}
$$

where $C(|u|,|v|)$ is bounded for $|u|,|v|$ bounded. We assume that $\inf _{u} F(x, u)=0$ $\forall x \in[0, L]$. Since we can add any function of $x$ to $F$ not changing $\nabla F$, this is not a restriction.

We denote by $H$ the Hilbert space $L_{2}(\Omega) \times L_{2}(\Omega), \Omega=(0, L) ; H_{1}$ is the Sobolev space of functions $u=\left(u_{1}, u_{2}\right)$ which satisfy $(1.2)$ with the bounded norm

$$
\|u\|_{1}=\left(\left\|\partial_{x} u\right\|^{2}+\|u\|^{2}\right)^{1 / 2}
$$

where $\|u\|$ is the norm in $H$,

$$
\|u\|^{2}=\int_{\Omega}\left|u_{1}(x)\right|^{2}+\cdots+\left|u_{d}(x)\right|^{2} d x .
$$

The space $H_{2}$ consists of functions which satisfy (1.2) and $\partial_{x}^{2} u \in H$.

Theorem 1.1. Let $u_{0} \in H_{1}$. Then there exists unique solution $u(t)$ of (1.1), (1.2) which belongs to $L_{\infty}\left([0, T], H_{1}\right) \cap L_{2}\left([0, T], H_{2}\right)$ for any $T>0$.

Proof. Local (in $t$ ) existence on a segment $\left[0, T_{0}\right]$ where $T_{0}$ depends on $\left\|u_{0}\right\|_{1}$ and uniqueness of solutions can be easily proved and is well-known (see for example, $[\mathrm{H}, \mathrm{BV}, \mathrm{L}]$. To prove existence on $[0, T]$ for arbitrary $T$, we consider the energy functional

$$
\mathcal{P}(u)=\int_{\Omega} a / 2\left|\partial_{x} u\right|^{2}+F(u, x) d x .
$$

Multiplying the equation (1.1) by $\partial_{t} u$ and integrating in $\alpha$ and $t$, we obtain the equation

$$
\mathcal{P}\left(u\left(t_{0}\right)\right)+\int_{0}^{t_{0}}\left\|\partial_{t} u\right\|^{2} d t=\mathcal{P}(u(0))
$$

Justification of this formula can be done as in [BV]. We do not need to impose growth conditions on $F^{\prime}$ here thanks to the Sobolev embedding $C(\bar{\Omega}) \supset H_{1}(\Omega)$ which holds in the one-dimensional case $(\Omega=(0, L))$.

From (1.6) we deduce

$$
\mathcal{P}\left(u\left(t_{0}\right)\right) \leq \mathcal{P}(u(0))
$$

when $0 \leq t_{0} \leq T_{0}$. Since

$$
\|u\|_{1}^{2} \leq \frac{2}{a} \mathcal{P}(u)+\|u\|^{2},
$$

the inequality (1.7) and the local existence imply in a usual way by the Gronwall inequality that the solution $u(t)$ exists for $0 \leq t<+\infty$ and satisfies (1.7) for all $t_{0}>0$.

Theorem 1.2. The problem (1.1), (1.2) generates a semigroup of operators $S_{t}$ : $H_{1} \rightarrow H_{1}$. These operators are continuous for $t \geq 0$ and are compact on bounded sets for $t>0$. 
Proof. Continuity of $S_{t}$ easily follows from the Lipschitz condition (1.4) and the boundedness of $u(t), t \in[0, T]$, in $C(\Omega)$ which follows from (1.7), (1.8) and the embedding $H_{1}(\Omega) \subset C(\Omega)$. Note that the mapping $u_{0} \rightarrow u(t)$ is continuous from $H_{1}$ into the space $W(0, T)$ with the norm defined by

$$
\|u\|_{W}^{2}=\int_{0}^{T}\|u\|_{2}^{2}+\left\|\partial_{t} u\right\|^{2} d t
$$

The mapping $u(t) \rightarrow F^{\prime}(u(t), x)$ is compact from $W(0, T)$ into $L_{2}([0, T], H)$. We consider (1.1) as a linear equation with $F^{\prime}$ a given function and an initial data $u_{0}$. Its solution $u(t) \in H_{1}$ with $t>0$ fixed depends completely continuously on $u(0) \in H_{1}$, and continuously on $F^{\prime}(u(t), \cdot) \in L_{2}([0, T], H)$. Therefore, it depends completely continuously on $u(0)$, and the theorem is proved.

Let us fix $\beta>0$ and consider the set $E_{\beta} \subset H_{1}$,

$$
E_{\beta}=\left\{v \in H_{1}: \mathcal{P}(v) \leq \beta\right\} .
$$

According to (1.7), the set $E_{\beta}$ is invariant with respect to $S_{t}$. Let $\left\{S_{t}\right\}$ be the semigroup generated by (1.1), (1.2a).

Theorem 1.3. For every $\beta>0$ there exists a global attractor $\mathcal{A}_{\beta} \subset E_{\beta}$ of the semigroup $\left\{S_{t}\right\}$ restricted to $E_{\beta}$.

Proof. The existence of $\mathcal{A}_{\beta}$ follows from boundedness of $E_{\beta}$ in $H_{1}$, compactness of $S_{t}$ and continuity of $S_{t}$ (see [BV, Ha]).

Note, that $\mathcal{A}_{\beta}$ may be a non-connected set. In fact, we shall show that under appropriate conditions on $F$ there exists many disjoint components of $\mathcal{A}_{\beta}$.

In the case of periodic boundary conditions we have to impose additional conditions to provide boundedness of $F_{\beta}$ :

$$
F(u) \rightarrow+\infty \quad \text { as }|u| \rightarrow \infty .
$$

Theorem 1.4. Let (1.10) hold in addition to conditions (1.3), (1.4). Then for any $\beta>0$ the semigroup $\left\{S_{t}\right\}$ generated by (1.1), (1.2b) has a global attractor $\mathcal{A}_{\beta} \subset E_{\beta}$.

Proof. The proof is the same as the one of Theorem 1.3 if we have boundedness of $E_{\beta}$ in $H_{1}$. To prove this boundedness, it suffices to show that the condition $\mathcal{P}(u) \leq \beta$ implies

$$
\left|\int_{0}^{L} u(x) d x\right| \leq \mathcal{C}
$$

for a constant $\mathcal{C}=\mathcal{C}(\beta)$. Assume that (1.11) were not true. Then we have a sequence $u_{j}$,

$$
\int_{0}^{L} u_{j}(x) d x=\mathcal{C}_{j}, \quad\left|\mathcal{C}_{j}\right| \rightarrow+\infty, \quad \mathcal{P}\left(u_{j}\right) \leq \beta .
$$

Condition (1.10) implies that

$$
F(u) \geq F_{0}(|u|), \quad F_{0}(|u|) \rightarrow \infty \quad \text { as }|u| \rightarrow \infty .
$$

Let

$$
v_{j}=u_{j}-\frac{1}{L} \int_{0}^{L} u_{j} d x
$$


We have

$$
\int_{0}^{L}\left|\partial_{x} v_{j}\right|^{2} d x \leq \beta, \quad \int_{0}^{L} v_{j} d x=0 .
$$

This implies compactness of $v_{j}$ in $\mathcal{C}([0, L])$, and after relabeling we may assume that $v_{j} \rightarrow v$ in $\mathcal{C}([0, L])$. Obviously,

$$
\int_{0}^{L} F\left(u_{j}\right) d x=\int_{0}^{L} F\left(v_{j}-\mathcal{C}_{j} / L\right) d x .
$$

Since $v_{j}$ are bounded in $\mathcal{C}([0, L])$,

$$
\left|v_{j}-\mathcal{C}_{j} / L\right| \geq\left|\mathcal{C}_{j}\right| / L-\left|v_{j}\right| \rightarrow \infty
$$

as $j \rightarrow \infty$ uniformly in $x$. This implies by (1.10)

$$
\int_{0}^{L} F\left(u_{j}\right) d x \rightarrow \infty \quad \text { as } j \rightarrow \infty
$$

and this contradicts the condition $\mathcal{P}\left(u_{j}\right) \leq \beta$. Hence, (1.11) is true. Boundedness of $\int_{0}^{L}\left|\partial_{x} u\right|^{2} d x,\left|\int_{0}^{L} u d x\right|^{2}$ implies boundedness of $u$ in $H_{1}([0, L])$, therefore $E_{\beta}$ is bounded. This implies existence of the attractor as in Theorem 1.3.

Remark 1.1. If the condition $F(u) u \geq \alpha|u|^{2}-C, \alpha>0$, is imposed, then (1.1) and (1.2) have a global attractor $\mathcal{A}$ (see $[\mathrm{BV}])$.

\section{Preservation of Homotopy Classes by the Semigroup}

In this section we give in case $d=2$ sufficient conditions on the potential $F$ under which the dynamics of $S_{t}$ on $E_{\beta}$ preserves classes of functions defined in homotopic terms. We consider in this and the following sections $x$-independent $F$ ( $x$-dependent $F$ will be considered elsewhere). More general situations and case $d>2$ are considered in Section 4 .

For a given finite set $P$ of points $P_{i}, i=1, \ldots, m$, let $d(P)=\inf _{i \neq j}\left|P_{i}-P_{j}\right|$. Let $\Omega(P, r)=\left\{y \in \mathbb{R}^{2}: \exists P_{i} \in P,\left|y-P_{i}\right| \leq r\right\}, \Omega^{\prime}=\mathbb{R}^{2} \backslash \Omega(P, r)=\Omega^{\prime}(P, r)$.

Consider the set $\mathcal{D}_{\mu} \subset \mathbb{R}^{2}$

$$
\mathcal{D}_{\mu}=\left\{y \in \mathbb{R}^{2}: F(y)<\mu\right\} .
$$

We assume that the potential $F$ and the value $\mu$ satisfy the following condition.

Condition 2.1. There exist points $P_{1}, \ldots, P_{m} \in \mathbb{R}^{2} \backslash b$ and numbers $r>0, M>0$ such that $r<d(P) / 2$ and

$$
F(y) \geq M \text { when } \operatorname{dist}(y, P) \leq r .
$$

Here and below

$$
\operatorname{dist}(y, P)=\inf _{i}\left\{\left|y-P_{i}\right|\right\} .
$$

We shall say that $C(P, r, M)$ holds if Condition 2.1 is fulfilled.

For a given set $\mathcal{D} \subset \mathbb{R}^{d}$ we denote by $\pi_{1}(\mathcal{D}, *)$ the fundamental homotopy group of classes of mappings from the circle $S^{1}$ into $\mathcal{D}$ (see [FFG, M, W]). Let $G=\pi_{1}\left(\Omega^{\prime}(P, r), *\right)$ be the fundamental homotopy group of $\Omega^{\prime}(P, r)$ (with a base point $*=b \in \Omega^{\prime}$ in the case of Dirichlet boundary conditions). Obviously, $G$ is independent on $r \geq 0$ if $r<d(P, M) / 2$ since $\Omega^{\prime}\left(P, r_{1}\right)$ is a deformation retract of $\Omega^{\prime}\left(P, r_{2}\right)$ when $0<r_{2} \leq r_{1}$. The set $\mathcal{D}_{M}^{\prime}=\mathbb{R}^{d} \backslash \mathcal{D}_{M}$ with large $M$ may be 
considered a (soft) barrier for curves $u(t)$. We can take $r_{0}$ as maximal $r$ such that $\Omega^{\prime}(P, r)$ is a deformation retract of $\mathcal{D}_{\mu}^{\prime}$. Obviously, $\Omega^{\prime}(P, r)$ is homotopy equivalent to a bunch of circles $\bigvee_{j=1}^{m} S_{j}^{1}$ and elements of $G$ can be uniquely represented in the form

$$
g=\prod_{j=1}^{n} g_{i_{j}}^{k_{j}}
$$

where $g_{i}$ is a generator of $j$-th group $\pi_{1}\left(S_{j}^{1}, *\right), k_{j} \in \mathbb{Z}, k_{j} \neq 0, i_{j} \neq i_{j+1}$. (In periodic case when we do not fix $*$, the representation is unique modulo cyclic permutations if $i_{1} \neq i_{n}$.) We denote deg $g=\left|k_{1}\right|+\cdots+\left|k_{m}\right|$.

Every continuous function $v(x), x \in[0, L]$, which satisfies (1.2a) and takes values in $\Omega^{\prime}(P, r)$ for all $x \in[0, L]$ belongs to a unique class of equivalence which is represented by $g=g(v) \in G$.

For $g \in G$, let $X\left(\Omega^{\prime}(P, r), g\right)$ be the set of $u \in C([0, \ell])$ which take values in $\Omega^{\prime}(P, r)$ and belong to $g$. Let

$$
Y(P, r, g, \beta)=X\left(\Omega^{\prime}(P, r), g\right) \cap E_{\beta} .
$$

(Note that by Sobolev embedding theorems $E_{\beta} \subset H_{1}[0, L] \subset \mathcal{C}([0, L])$.) We shall prove below that under appropriate conditions on $F$ and $\beta$ the set $Y(P, g, \beta)$ is invariant with respect to $S_{t}$. Moreover, there exists $\delta>0$ such that

$$
S_{t}(Y(P, 0, g, \beta)) \subset Y(P, \delta, g, \beta) \quad \forall t \geq 0, \quad \forall g \in G .
$$

(Note that the empty set is always invariant, $S_{t} \emptyset=\emptyset$.) We shall for brevity denote $Y(g, \beta)=Y(P, 0, g, \beta)$.

Theorem 2.1. Let Dirichlet boundary condition (1.2a) hold, $R=\min _{j}\left|P_{j}-b\right|$. Let Condition (1.2a) hold. Let $r>\delta>0, r \leq R$ and

$$
\beta<\sqrt{8}(r-\delta) \sqrt{a} \sqrt{M}+\frac{2(R-r)^{2} a}{L} .
$$

Then for any $u \in E_{\beta}$

$$
\operatorname{dist}(u(x), P)>\delta, \quad \forall x \in[0, L] .
$$

Here and below $\operatorname{dist}(v, P)=\inf _{z \in P}|v-z|$.

Proof. To prove (2.7), assume the contrary, that is

$$
\exists P_{1} \in P, \quad\left|u\left(x_{0}\right)-P_{1}\right|=\delta, \quad x_{0} \in(0, L) .
$$

Let $r_{1}=r-\delta$. Changing variable $v=u-P_{1}$, we obtain from (2.8) that

$$
v\left(x_{0}\right)=P_{0}, \quad\left|P_{0}\right|=\delta, \quad v(0)=v(L)=b-P_{1} .
$$

We choose such $x_{0}=x^{*}$ that is minimal over all possible $x_{0}$ which satisfy $(2.8), x_{*}$ being the maximal of such $x_{0}$. Let

$$
U(\rho)=\inf _{|v| \leq \rho} F\left(P_{1}+v\right) .
$$

Consider $\mathcal{P}(u)$,

$$
\mathcal{P}(u)=\int_{0}^{L} a / 2\left|\partial_{x} u\right|^{2}+F(u) d x=\int_{0}^{L} a / 2\left|\partial_{x} v\right|^{2}+F\left(P_{1}+v\right) d x .
$$


Obviously $v(x) \neq 0$ for $x \in\left(0, x^{*}\right) \cup\left(x_{*}, L\right)$. Taking (2.10) into account, we see that

$$
\mathcal{P}(u) \geq \int_{0}^{x^{*}} a / 2\left|\partial_{x} v\right|^{2}+U(|v|) d x+\int_{x_{*}}^{L} a / 2\left|\partial_{x} v\right|+U(|v|) d x .
$$

Using polar coordinates $(\rho, \phi)$ in $\mathbb{R}^{2}, \rho=|u|$, we see that

$$
\left|\partial_{x} v\right|^{2}=\left|\partial_{x} \rho\right|^{2}+\rho^{2}\left|\partial_{x} \phi\right|^{2} .
$$

Therefore,

$$
\mathcal{P}(u) \geq \int_{0}^{x_{0}^{*}} a / 2\left|\partial_{x}\right| v||^{2}+U(|v|) d x+\int_{x_{*}}^{L} a / 2\left|\partial_{x}\right| v \|^{2}+U(|v|) d x .
$$

Let

$$
\begin{aligned}
& U^{*}(\rho)=M \text { when } 0 \leq \rho \leq r, \\
& U^{*}(\rho)=0 \text { when } \rho>r .
\end{aligned}
$$

Since Condition 2.1 holds,

$$
U(|v|) \geq U^{*}(|v|)
$$

and we have

$$
\mathcal{P}(u) \geq \int_{0}^{x^{*}} a / 2\left|\partial_{x}\right| v||^{2}+U^{*}(|v|) d x+\int_{x_{*}}^{L} a / 2\left|\partial_{x}\right| v||^{2}+U^{*}(|v|) d x .
$$

We apply Lemma 2.1 (see below) where $\ell=x^{*}$, and $r-\delta=r_{1}, R_{1}=R-\delta$. We obtain:

$$
\int_{0}^{x^{*}} a / 2\left|\partial_{x}\right| v||^{2}+U^{*}(|v|) d x \geq \sqrt{2} r_{1} \sqrt{a} \sqrt{M}+\frac{\left(R_{1}-r_{1}\right)^{2} a}{2 x^{*}} .
$$

Analogously,

$$
\int_{x_{*}}^{L} a / 2\left|\partial_{x}\right| v||^{2}+U^{*}(|v|) d x \geq \sqrt{2} r_{1} \sqrt{a M}+\frac{\left(R_{1}-r_{1}\right)^{2} a}{2\left(L-x_{*}\right)} .
$$

These inequalities and (2.6) imply that $\mathcal{P}(u)>\beta$, which contradicts the assumption $u \in E_{\beta}$. Therefore (2.7) holds, and the theorem is proved.

Lemma 2.1. Let $U^{*}(\rho)$ be defined by (2.12). Let $w \in H_{1}([0, \ell])$ be a scalar function, $w(0)=0, w(\ell)=R_{1} \geq r_{1}>0$. Then

$$
\mathcal{P}\left(w, U^{*}\right)=\int_{0}^{\ell} a / 2\left|\partial_{x} w\right|^{2}+U^{*}(|w|) d x \geq \sqrt{2} r_{1} \sqrt{a} \sqrt{M}+\frac{\left(R_{1}-r_{1}\right)^{2} a}{2 \ell} .
$$

Proof. We take $R_{1}>r_{1}$. Obviously, we can find a function $U_{\epsilon} \in C^{2}(\mathbb{R}), U_{\epsilon}(\rho) \leq$ $U^{*}(\rho) \forall \rho, U_{\epsilon}(\rho)=M$ when $\rho \leq r_{1}-\epsilon, U_{\epsilon}(\rho)=0$ when $\rho \geq r_{1}, U_{\epsilon}^{\prime}(\rho) \leq 0$, $U_{\epsilon}(\rho) \rightarrow U^{*}(\rho)$ when $\epsilon \rightarrow 0$. Let $w_{\epsilon}=w$ be a global minimizer of $\mathcal{P}^{*}\left(v, U_{\epsilon}\right)$. Such a minimizer exists. This easily follows from boundedness of the set $P(u) \leq \epsilon$ in $M_{1}([0, \ell])$, compactness of the embedding $M_{1}([0, \ell]) \subset \mathcal{C}([0, \ell])$ and continuity of $U_{\epsilon}$. One also has to use bilinearity and positiveness of the main part of $P(v)$. This function $w$ is a smooth solution of the Euler equation

$$
-a \partial_{x}^{2} w+U_{\epsilon}^{\prime}(w)=0, \quad w(0)=0, \quad w(\ell)=R_{1} .
$$

This equation admits the integral

$$
-a / 2\left(\partial_{x} w\right)^{2}+U_{\epsilon}(w)=-E .
$$


Since $U_{\epsilon}^{\prime}<0, R_{1}>r_{1}$, one can easily see that $w$ is a convex growing function, and $v$ is linear for $0<x<x_{1}$ when $w<r_{1}-\epsilon$ and for $x \geq x_{2}$ when $w \geq r_{1}$. We obtain that

$$
\begin{aligned}
& w=x \sqrt{2(E+M)} / \sqrt{a} \quad \text { when } w \leq r_{1}-\epsilon, \\
& w=R+\sqrt{2 E}(x-\ell) \sqrt{a} \quad \text { when } \ell \geq w \geq r_{1} .
\end{aligned}
$$

We have also

$$
\frac{\sqrt{2 E}}{\sqrt{a}}\left(\ell-x_{2}\right)=R_{1}-r_{1}, \quad \frac{x_{1} \sqrt{2(E+M)}}{\sqrt{a}}=r_{1}-\epsilon .
$$

We see that

$$
\begin{aligned}
\int_{0}^{\ell} a / 2\left|\partial_{x} w\right|^{2}+U_{\epsilon}(w) d x & \geq x_{1}(E+M)+x_{1} M+E\left(\ell-x_{2}\right) \\
& =\frac{a\left(r_{1}-\epsilon\right)^{2}}{2 x_{1}}+x_{1} M+\frac{\left(R_{1}-r_{1}\right)^{2} a}{2\left(\ell-x_{2}\right)} \\
& \geq \sqrt{2}\left(r_{1}-\epsilon\right) \sqrt{a} \sqrt{M}+\frac{\left(R_{1}-r_{1}\right)^{2} a}{2 \ell} .
\end{aligned}
$$

Hence, $\mathcal{P}^{*}\left(v, U^{*}\right) \geq \mathcal{P}^{*}\left(v, U_{\epsilon}\right) \geq \sqrt{2}\left(r_{1}-\epsilon\right) \sqrt{M} \sqrt{a}+\left(R_{1}-r_{1}\right)^{2} a /(2 \ell)$. Since $\epsilon$ is arbitrarily small, we obtain (2.13). If $R_{1}=r_{1}$, we can pass in (2.13) to the limit as $R_{1} \rightarrow r_{1}$.

Remark. In fact, from the proof of Lemma 2.1, we see that we have the inequality

$$
\mathcal{P}\left(w, U^{*}\right) \geq \min _{0 \leq x_{1} \leq \ell} \frac{a r_{1}^{2}}{2 x_{1}}+x_{1} M+\frac{\left(R_{1}-r_{1}\right)^{2} a}{2\left(\ell-x_{1}\right)} .
$$

The inequality (2.13) gives a convenient lower estimate of the right-hand side. If $R_{1} \leq r_{1}$, we have $\mathcal{P}\left(w, U^{*}\right) \geq r_{1} \sqrt{2 M a}$.

Corollary 2.1. Under conditions of Theorem 2.1, (2.5) holds.

Proof. By (1.7) $S_{t} E_{\beta} \subset E_{\beta}$. Since $u(x, t)$ is continuous in $x, t$, two curves $u\left(x, t_{1}\right)$, $u\left(x, t_{2}\right)$ are homotopy equivalent. Using (2.7), we obtain (2.5).

Theorem 2.2. Under conditions of Theorem 2.1, the semigroup $\left\{S_{t}\right\}$ restricted to $Y(g, \beta)$ has the attractor $\mathcal{A}(g, \beta) \subset Y(g, \beta)$. The attractor $\mathcal{A}_{\beta}$ can be represented in the form

$$
\mathcal{A}=\bigcup_{g \in G} \mathcal{A}(g, \beta)
$$

where $\mathcal{A}(g, \beta)$ are disjoint sets. If $Y(g, \beta)$ is not empty, $\mathcal{A}(g, \beta)$ is not empty. $\mathcal{A}(g, \beta)$ is stable and contains a stable set $\mathcal{N}_{0}(\mathcal{A}(g, \beta))$ of $t$-independent solutions of (1.1), (1.2), it includes a connected component $X \subset \mathcal{N}_{0}(\mathcal{A}(g, \beta))$.

Proof. Since $E_{\beta} \cap g$ is invariant by Corollary 2.1, the existence of $\mathcal{A}(g, \beta)$ is proved exactly like the existence of $\mathcal{A}(\beta)$. By $(2.7)$,

$$
E_{\beta}=\bigcup_{g} Y(g, \beta) .
$$

By Theorem 1.1 there exists $\delta>0$ such that

$$
\left|u(x)-P_{j}\right| \geq \delta>0 \quad \forall x \in L, \quad P_{j} \in P, \quad u \in E_{\beta} .
$$


From (2.17) it follows that every $Y(g, \beta)$ is open and closed in $H_{1} \cap E_{\beta}$. Therefore $\mathcal{A}(g, \beta), \mathcal{A}\left(g^{\prime}, \beta\right)$ lie at a finite distance if $g \neq g^{\prime}, \mathcal{A}(g, \beta) \neq \emptyset, \mathcal{A}\left(g^{\prime}, \beta\right) \neq \emptyset . \mathcal{A}(g, \beta)$ is stable, see $[\mathrm{BV}]$. Let

$$
\underline{\mathcal{P}}(g, \beta)=\inf _{u \in \mathcal{A}(g, \beta)} \mathcal{P}(u) .
$$

Since $\mathcal{A}(g, \beta)$ is compact, for non-empty $\mathcal{A}(g, \beta)$ the set

$$
\mathcal{N}(g, \beta)=\{u \in \mathcal{A}(g, \beta): \mathcal{P}(u)=\underline{\mathcal{P}}(g, \beta)\}
$$

is non-empty and compact. Let $X=\mathcal{N}(g, \beta)$. First, $X$ consists of equilibrium points of $\left\{S_{t}\right\}$. Let $\sigma(X)$ be a small neighborhood of $\mathcal{A}$. Indeed, if $u_{0} \in \sigma(X) \subset$ $Y(g, \beta), u(t)=S_{t} u_{0}$ is attracted to $\mathcal{A}(g, \beta)$. We have

$$
\mathcal{P}(u(t)) \leq \mathcal{P}\left(u_{0}\right) .
$$

Let $Y^{\prime}=\operatorname{cl}\left[S_{1}(Y(a, \beta))\right]$ be the closure in $H_{1}([0, L])$, obviously (see Theorem 1.2) $Y$ is a compact invariant subset of $Y(g, \beta)$. Let $Y^{\prime}(\gamma)=Y^{\prime} \cap\{u: \mathcal{P}(u)=\gamma\}$,

$$
\mathcal{P}_{1}=\sup _{u \in Y^{\prime}(\gamma)} \mathcal{P}(u), \quad \mathcal{P}_{2}=\inf _{u \in Y^{\prime}(\gamma)} \mathcal{P}(u) .
$$

Since $\mathcal{P}\left(S_{t} u\right)$ is a decreasing function of $\gamma$ and $\mathcal{A}(\gamma, \beta)$ is the attractor, we have

$$
\mathcal{P}_{1}=\underline{\mathcal{P}}(g, \beta) .
$$

The set $Y^{\prime}\left(\mathcal{P}_{2}\right)$ consists of equilibrium points of $S_{t}$, since $\mathcal{P}\left(S_{t} u\right)$ is a strictly decreasing function of $t$ on non-equilibrium points.

The sets $Y^{\prime}(\gamma)$ upper semicontinuously depend on $\gamma$ (this follows from compactness of $Y^{\prime}$ and continuity of $\mathcal{P}$ ). Therefore, if $\sigma$ is a given neighborhood of $Y^{\prime}\left(\mathcal{P}_{2}\right)$, there exists $\delta>0$ such that $Y^{\prime}(\gamma) \subset \sigma$ when $\left|\gamma-\mathcal{P}_{2}\right| \leq \delta$. Since the set $\left\{u \in Y^{\prime}: \mathcal{P}(u) \leq \mathcal{P}_{2}+\delta\right\}$ is invariant, this implies stability of $Y^{\prime}\left(\mathcal{P}_{2}\right)$. Using the fact that a trajectory $u(t)$ is a continuous function of $t$, we easily conclude that every (maximal connected) component $X$ of $Y^{\prime}\left(\mathcal{P}_{2}\right)$ is stable, and Theorem 2.2 is proved.

Definition 2.1. For a set $\mathcal{D} \subset \mathbb{R}^{2}, * \in \mathcal{D}$ let $\mathcal{L}(\mathcal{D}, *)$ be the set of functions $u(*)$, $u:[0, L] \rightarrow \mathcal{D}, u(0)=u(L)=*$, such that $u \in H_{1}([0, L])$.

Remark 2.2. Since $H_{1}([0, L]) \subset \mathcal{C}([0, L])$ by Sobolev's embedding theorem, any $u \in \mathcal{L}(\mathcal{D}, *)$ is continuous and belongs to a class $g \in \pi_{1}(\mathcal{D}, *)$. (As usual, we denote by $\pi_{1}(\mathcal{D}, *)$ the fundamental homotopy group of $\mathcal{D}$ with a base point *.) Moreover, since the arc length $\ell(u)$ of the curve is estimated by

$$
\ell(u)=\int_{0}^{L}\left|\partial_{x} u\right| d x \leq \sqrt{L}\left(\int_{0}^{L}\left|\partial_{x} u\right|^{2} d x\right)^{1 / 2},
$$

any $u \in \mathcal{L}(\mathcal{D}, *)$ has a finite length $\ell(u)$.

Definition 2.2. For a set $\mathcal{D} \subset \mathbb{R}^{2}, * \in \mathcal{D}$ any $g \in \pi_{1}(\mathcal{D}, *)$ let

$$
\ell(g, \mathcal{D})=\inf _{u \in g \cap \mathcal{L}(\mathcal{D}, *)} \ell(u) .
$$

Remark 2.3. Obviously, if $* \in \mathcal{D}^{\prime} \subset \mathcal{D}$ and $\mathcal{D}^{\prime}$ is a deformation retract of $\mathcal{D}$, then $\pi_{1}\left(\mathcal{D}^{\prime}, *\right)=\pi_{1}(\mathcal{D}, *)$ and $\ell\left(g, \mathcal{D}^{\prime}\right) \geq \ell(g, \mathcal{D})$. 
Lemma 2.2. Let $g \in \pi_{1}(\mathcal{D}, *)$. If $\ell(g, \mathcal{D})<\infty$, then for any $\epsilon>0$ there exists $u \in \mathcal{L}(\mathcal{D}, *) \cap g$ such that

$$
\mathcal{P}(u) \leq L \mu(\mathcal{D}, F)+\frac{a}{2 L}(\ell(g, \mu))^{2}+\epsilon
$$

where

$$
\mu(\mathcal{D}, F)=\sup _{u \in \mathcal{D}} F(u)
$$

Proof. According to the definition of $\ell(g, \mathcal{D})$ for any $\delta>0$ there exists $u \in \mathcal{L}(\mathcal{D}, *)$ such that

$$
\ell(u) \leq \ell(g, \mathcal{D})+\delta
$$

Let $s(y)$ be the length of the arc of the curve $u(x), 0 \leq x \leq y, y \leq L$,

$$
s(y)=\int_{0}^{y}\left|\partial_{x} u(\theta)\right| d \theta .
$$

Let $y(s), 0 \leq s \leq \ell(u)$, be the inverse function. The function $\tilde{u}(x)=u\left(y\left(x \frac{\ell(u)}{L}\right)\right)$ belongs to $\mathcal{L}(\mathcal{D}, *)$. We have

Since

$$
\partial_{x} \tilde{u}(x)=u^{\prime} \cdot y^{\prime} \cdot \frac{\ell(u)}{L} .
$$

$$
u^{\prime}(y) \cdot y^{\prime}(s)=u^{\prime}(y) / s^{\prime}(y)=1
$$

we obtain

$$
\partial_{x} \tilde{u}(x)=\ell(u) / L
$$

We have

$$
\begin{aligned}
\mathcal{P}(\tilde{u}) & =\int_{0}^{L}\left[\frac{a}{2}\left|\partial_{x} \tilde{u}\right|^{2}+F(\tilde{u})\right] d x \\
& \leq L \mu(\mathcal{D}, F)+\frac{a}{2 L} \ell(u)^{2} \leq L \mu(\mathcal{D}, F)+\frac{a(\ell(g, \mathcal{D})+\delta)^{2}}{2 L} .
\end{aligned}
$$

This implies (2.17b).

Remark 2.4. If $\mathcal{D}$ is closed and its boundary is piecewise smooth, $\ell(g, \mathcal{D})=\ell\left(u_{0}\right)$ where $u_{0}(x)$ is a geodesic curve which belongs to the class $g$. Obviously, $u_{0}(x)$ consists of line segments and arcs which belong to $\partial \mathcal{D}$.

Remark 2.5. If $* \in \mathcal{D}^{\prime} \subset \mathcal{D}$, then the natural mapping $\mathcal{T}: \pi_{1}\left(\mathcal{D}^{\prime}, *\right) \rightarrow \pi_{1}(\mathcal{D}, *)$ is well-defined. If $\mathcal{D}^{\prime}$ is a deformation retract of $\mathcal{D}$, this mapping is isomorphism, and we identify $g$ and $\mathcal{T} g$.

Theorem 2.3. Let $\mu$ be such that the domain $\mathcal{D}_{\mu}$ defined by $(2.1)$ lies in $\Omega^{\prime}(P, r)$ with $r>0$ and $\mathcal{D}_{\mu}$ is a deformation retract of $\Omega^{\prime}(P, r)$. Let $v_{i}:[0, L] \rightarrow \mathcal{D}_{\mu}$ be $C^{1}$ mappings which represent the generators $g_{i}$ of the homotopy group $G=\pi\left(\mathcal{D}_{\mu}\right)=$ $\pi\left(\Omega^{\prime}(P, 0), *\right)$ and the curves $v_{i}$ have finite lengths. Let the lengths of the curves $v_{i}(x)$ be equal to $\ell_{i}$. Let $g \in G$ be defined by 2.3). Then there exists $u \in X\left(\Omega^{\prime}(P, r)\right)$ such that $u \in E_{\beta}$,

$$
\beta \leq L \mu+\frac{a}{2 L}\left(\sum_{j=1}^{n}\left|k_{j}\right| \ell_{i_{j}}\right)^{2}
$$

where $k_{j}$ are the same as in (2.3) 
Proof. Let $s(y)$ be the length of the curve $v_{i}(x)$ between 0 and $v_{i}(y)$,

$$
s_{i}(y)=\int_{0}^{y}\left|\partial_{x} v_{i}^{\prime}(\theta)\right| d \theta .
$$

Let $y_{i}(s)$ be the inverse of $s_{i}(y)$, let

$$
\tilde{v}_{i}(s)=v_{i}\left(y_{i}(s)\right), \quad 0 \leq s \leq \ell_{i} .
$$

Let

$$
\bar{\ell}=\sum_{j=1}^{n}\left|k_{j}\right| \ell_{i_{j}}
$$

be the total length of the curve corresponding to $g$, defined by (2.3). The segment $[0, \bar{\ell}]$ is covered by $N=\operatorname{deg} g=\left|k_{1}\right|+\cdots+\left|k_{n}\right|$ segments of length $\ell_{i_{j}}$ in the order determined by $i_{j},\left|k_{j}\right|$. We denote the end points of these segments by $x_{j}, j=0$, $N$. $x_{p}$ may be explicitly written as

$$
x_{p}=\sum_{j=1}^{\sigma-1}\left|k_{j}\right| \ell_{i_{j}}+k \ell_{i \sigma}, \quad p=\sum_{j=1}^{\sigma-1}\left|k_{j}\right|+k,
$$

where $0 \leq k \leq\left|k_{\sigma}\right|$. Obviously, $g_{i \sigma}$ is at $p$-th position in the product (2.3), the function $i=i(p), 1 \leq i \leq m$, is well defined, $i(p)$ is the number of the generator $g_{i}$ which is at $p$-th position in (2.3). We put

$$
u\left(x_{p}+x\right)=\tilde{v}_{i(p)}(x), \quad 0 \leq x \leq \ell_{i(p)} .
$$

The function $u(x), 0 \leq x \leq \bar{\ell}$, is continuous for all $x$ and continuously differentiable at every point $x \neq x_{q}, q=0, \ldots, N$.

Let $\bar{u}(x)$ be $u(x \bar{\ell} / L), 0 \leq x \leq L$. This function represents the homotopy class $g \in G$ (see, for example [FFG, M, W]).

Obviously, $\left|\partial_{x} u\right|=\bar{\ell} / L$ at $x \neq x_{p}$. Computing $\mathcal{P}(u)$, we obtain

$$
\mathcal{P}(u)=\frac{a}{2} L^{-1} \bar{\ell}^{2}+\mu L=\frac{a}{2} L^{-1}\left(\sum_{j=1}^{n}\left|k_{j}\right| \ell_{i_{j}}\right)^{2}+\mu L .
$$

This implies (2.18).

Theorem 2.4. Let periodic boundary condition (1.2b) hold. Let $g$ be defined by (2.3) with $n \geq 2$. Let (2.6) hold with $R=d(P)$. Then (2.5) holds.

Proof. We repeat literally the proofs of Theorem 2.1 and Corollary 2.1. The only difference is that instead of (2.9) we have

$$
v\left(x_{0}\right)=P_{0}, \quad\left|P_{0}\right|=\delta, \quad\left|v\left(x_{1}\right)\right| \geq R
$$

where $x_{1} \in[0, L], x_{0} \in[0, L]$. We have (2.19) because otherwise $v\left(x_{1}\right) \leq d(P) \forall$ $x_{1} \in[0, L]$ and the curve $u(x)$ is homotopic to an element $g_{1}^{k_{1}}$.

Remark 2.6. In the case $g=g_{1}^{k_{1}}$ the following inclusion holds:

$$
S_{t}(Y(P, r, g, \beta)) \subset Y(P, \delta, g, \beta) \quad \forall t \geq 0
$$

if $\beta<r \sqrt{M a} / 2$. (For the proof see Theorem 4.2.) 
Remark 2.7. One can easily see that the function constructed in Theorem 2.3 satisfies periodic boundary conditions as well, since functions from $H_{1}$ admit discontinuous derivatives.

Theorem 2.5. Let $b \in \mathcal{D} \subset \mathcal{D}_{M}=\{u: F(u) \leq M\}$. Let $\Omega(P, r) \subset \mathbb{R}^{2} \backslash \mathcal{D}_{M}$, $M>\mu_{0}$. Let $g \in \pi_{1}(\mathcal{D}, *)$. Let

$$
L \mu(\mathcal{D}, F)+\frac{a \ell(g, \mathcal{D})^{2}}{2 L}<\sqrt{8} r \sqrt{M a}+\frac{2(R-r)^{2}}{L},
$$

$\mu(\mathcal{D}, F)$ is the same as in Lemma 2.2. Let $g_{*}=\mathcal{T}_{*} g$, where $\mathcal{T}: \pi_{1}(\mathcal{D}, *) \rightarrow$ $\pi_{1}\left(\Omega^{\prime}(P, r), *\right)$ is the homomorphism generated by the embedding $\mathcal{D} \subset \Omega^{\prime}(P, r)=$ $\mathbb{R}^{2} \backslash \Omega(P, r)$. Then there exists such $\beta>0$ that the component $\mathcal{A}\left(\mathcal{T}_{*} g, \beta\right)$ of the global attractor $\mathcal{A}$ is not empty, $\mathcal{A}\left(\mathcal{T}_{*} g, \beta\right) \neq \emptyset$.

Proof. By (2.20) we can find such $\beta$ and $\delta_{1}, \delta_{2}>0$ that

$$
\begin{gathered}
L \mu(\mathcal{D}, F)+\frac{a \ell(g, \mathcal{D})^{2}}{2 L}<\beta-\delta_{1}, \\
\beta<\sqrt{8}\left(r-\delta_{2}\right) \sqrt{M a}+\frac{2(R-r)^{2}}{L} .
\end{gathered}
$$

By Lemma 2.2 there exists $u \in g$ such that $\mathcal{P}(u)<\beta$, therefore $u \in E_{\beta} \cap$ $X\left(\Omega^{\prime}(P, r), \mathcal{T}_{*} g\right)=Y\left(\mathcal{T}_{*} g, \beta\right)$. By Theorem 2.1 and Corollary 2.1 we have $(2.5)$ with $g$ replaced by $\mathcal{T}_{*} g$. By Theorem 2.2 we obtain that $\mathcal{A}\left(\mathcal{T}_{*} g, \beta\right) \neq \emptyset$ and the theorem is proved.

Theorem 2.6. Let $\delta>0$, let $\beta$ satisfy (2.6). Then the mapping

$$
\mathcal{B}: u \rightarrow g(u)
$$

where $g=g(u) \in \pi_{1}\left(\Omega^{\prime}(P, 0, g), *\right)$ is the homotopy class of $u$, is well-defined. This mapping is continuous on $E_{\beta}$.

Proof. The first statement directly follows from Theorem 2.1 with $t=0, \delta=0$. To obtain the second statement note that if $\|u-\tilde{u}\|_{1}<\epsilon$, then by Sobolev embedding theorem

$$
|u(x)-\tilde{u}(x)| \leq \epsilon \quad \forall x \in[0, L]
$$

Consider

$$
u_{\tau}=(1-\tau) u+\tau \tilde{u}, \quad \tau \in[0,1]
$$

If $\epsilon$ is small enough,

$$
u_{\tau}(x) \in \Omega^{\prime}(P, \delta-\epsilon, g) \quad \forall x \in[0, L] .
$$

At the same time, since the functional $\mathcal{P}$ is continuous on $H_{1}([0, L])$, (see $\left.[\mathrm{BV}]\right)$, $\mathcal{P}\left(u_{\delta}\right)$ depends continuously on $\tau$, and

$$
\mathcal{P}\left(u_{\tau}\right) \leq \beta+C \epsilon \quad \text { when } \tau \in[0,1]
$$

Therefore, (2.7) holds with $\delta$ replaced by $\delta / 2$ if $\epsilon$ is small enough. Hence, by (2.5), $g\left(u_{\tau}\right)$ is well-defined (since $\Omega^{\prime}(P, \delta)$ is a deformation retract of $\Omega^{\prime}(P, \delta-\epsilon), g$ does not depend on $\delta$ ), and since $u_{\tau}$ is a homotopy, $g\left(u_{\tau}\right)=g(u)$. So, $g$ is continuous, and the theorem is proved.

Remark 2.8. The mapping $\mathcal{B}: E_{\beta} \rightarrow \pi_{1}\left(\Omega^{\prime}(P, r), *\right)$ generates decomposition of $E_{\beta}$ into disjoint basins $K_{g}, g \in G, K_{g}=\mathcal{B}^{-1}(g)$. 
Theorem 2.7. Let (2.6) hold. Then the mapping

$$
\mathcal{B}: E_{\beta} \rightarrow G
$$

maps $E_{\beta}$ onto subset $G_{1} \in G$, which consists of elements $g$ defined by (2.3) and satisfying

$$
\left(\sum\left|k_{j}\right| \ell_{i_{j}}\right)^{2} \leq \frac{2 L \beta}{a}-\frac{2 L^{2} \mu}{a} .
$$

Proof. By Theorem 2.3, if (2.21) holds, then there exists $u \in g \cap E_{\beta}$. That means $\mathcal{B}(u)=g$, and the theorem is proved.

\section{Examples of Existence of Complicated Spatial Structure of Attractors}

Now we give a simple example which illustrates Theorems $2.1-2.5$ and their corollaries. We identify the plane $\mathbb{R}^{2}$ with the complex plane $\mathbb{C}$.

Example 3.1. Consider the equations (1.1), (1.2a) with $a=1$ and the potential $F_{\epsilon}: \mathbb{C} \rightarrow \mathbb{R}$ which is defined by the formula

$$
F_{\epsilon}(u)=M_{1}\left|u-z_{1}\right|^{-p}+M_{1}\left|u-z_{2}\right|^{-p}
$$

for $\left|u-z_{i}\right| \geq \epsilon>0, p>2$. We assume that $F_{\epsilon}(u)$ belongs to $C^{2}\left(\mathbb{R}^{2}\right)$ and

$$
F_{\epsilon}(u) \geq M_{1} \epsilon^{-p} \quad \text { when }\left|u-z_{j}\right| \leq \epsilon .
$$

(This potential can be considered as a singular potential modified in the $\epsilon$-neighborhoods of singular points.) Here we take

$$
z_{1}=(-R, 0)=-R, \quad z_{2}=(R, 0)=R, \quad R \geq 2 .
$$

We take $P_{1}=z_{1}, P_{2}=z_{2}$ in notations of Section $2, P=\left\{P_{1}, P_{2}\right\}, m=2$. The set $\Omega^{\prime}(P, 1)$ is the plane with two deleted disks of radius 1 . Now we define the generators of the fundamental homotopy group $\pi_{1}\left(\Omega^{\prime}(P, 1)\right)$. The first generator $g_{1}$ coincides with the homotopy class of mappings $u: S^{1} \rightarrow \Omega^{\prime}(P, 1)$ (we put $S^{1}=[0,2 \pi]$ with points 0 and $2 \pi$ considered as the same point, it is the designated point $* \in S^{1}$ ) which map $[0,2 \pi]$ into a curve turning one time around $z_{1}$ in counter-clockwise direction. The fixed representative of this class is

$$
g_{10}(x)=z_{1}+R e^{i x} .
$$

Obviously, $g_{10}(*)=0,0$ coincides with the designated point $* \in \mathbb{R}^{2}=\mathbb{C}$. The generator $g_{2}$ corresponds to curves around $z_{2}$ in counter-clockwise direction, and is represented by

$$
g_{20}(x)=z_{2}-R e^{i x} .
$$

The group $G$ is not commutative and consists of all monomials generated by $g_{1}$ and $g_{2}$. For example, the monomial $g=g_{1}^{2} g_{2} g_{1}^{-1}$ of degree 4 corresponds to two counter-clockwise turns around $z_{1}$ followed by one counter-clockwise turn around $z_{2}$ and one clockwise turn around $z_{1}$. This element is represented by the function $g_{0}(x)$

$$
\begin{aligned}
& g_{0}(x)=z_{1}+\operatorname{Re}^{4 i x} \text { on }[0, \pi] ; \\
& g_{0}(x)=z_{2}-\operatorname{Re}^{4 i x} \text { on }[\pi, 3 \pi / 2] ; \\
& g_{0}(x)=z_{1}+\operatorname{Re}^{-4 i x} \text { on }[3 \pi / 2,2 \pi] .
\end{aligned}
$$


Now we give a condition on $r$ and $M$ which guarantees that Condition 2.1 holds. By (3.1) and (3.2) if $r \geq \epsilon$ we have

$$
F_{\epsilon}(u) \geq M_{1} r^{-p}
$$

when $\operatorname{dist}(u, P) \leq r \leq 1$ where $p>2$. Therefore Condition 2.1 holds with $M=r^{-p}$, for $1 \geq r \geq \epsilon$.

Now for given $\beta$ condition (2.6) of Theorem 2.1 is fulfilled if

$$
\beta \leq \sqrt{8}(r-\delta) r^{-p / 2} \sqrt{M_{1}}
$$

if $1>r>\epsilon$. Since $\delta>0$ is arbitrary, we can take $\delta=\epsilon / 2, r=\epsilon$. Theorem 2.1 implies

$$
S_{t}(Y(g, \beta)) \subset Y(g, \beta) \quad \forall t \geq 0
$$

if

$$
\beta \leq \epsilon^{1-p / 2} \sqrt{2 M_{1}}
$$

Therefore the sets $\mathcal{A}(g, \beta)$ and $\mathcal{N}(g, \beta)$ are well-defined for such $\beta$. Note that if $\mathcal{N}\left(g, \beta_{0}\right) \neq \emptyset$, then $\mathcal{N}(g, \beta)=\mathcal{N}\left(g, \beta_{0}\right)$ if $\beta \geq \beta_{0}$. Indeed, $\mathcal{A}(g, \beta) \supset \mathcal{A}\left(g, \beta_{0}\right)$ since $E\left(\beta_{0}\right) \subset E(\beta)$. Hence, $\mathcal{N}\left(g, \beta_{0}\right) \subset \mathcal{N}(g, \beta)$ for $\beta \geq \beta_{0}$. At the same time, by (2.17) $\mathcal{N}(g, \beta) \subset E(\underline{\beta}), \underline{\beta}=\underline{\mathcal{P}}(g, \beta)$ and since $\mathcal{N}\left(g, \beta_{0}\right) \subset \mathcal{A}(g, \beta)$, we have $\underline{\beta} \leq \beta_{0}$. This implies $\mathcal{N}(g, \bar{\beta}) \subset \mathcal{N}\left(g, \beta_{0}\right)$.

Consider now the question: for which $g$ are the sets $\mathcal{A}(g, \beta)$ and $\mathcal{N}(g, \beta)$ nonempty? A sufficient condition is given by Theorem 2.3. For a $g$ given by (2.3) (where we take $m=2$ ) and generators $g_{1}$ and $g_{2}$ defined above, we have a curve $u_{g} \in E_{\beta}, u_{g} \in g$ where

$$
\beta \leq L \mu+\frac{1}{2 L}\left(\sum_{j=1}^{n}\left|k_{j}\right| 2 \pi R\right)^{2} .
$$

(Obviously, for generators corresponding to $g_{10}, g_{20}$ defined by (3.3), (3.4) the lengths $\ell_{i}=2 \pi R$.) Obviously, $g_{10}$ and $g_{20}$ take their values in $\left\{F_{\epsilon}(u) \leq 2 R^{-p}\right\}$, therefore we can take

$$
\mu=2 \cdot R^{-p}
$$

Therefore, combining (3.8) and (3.9) we see that $\mathcal{N}(g, \beta) \neq \emptyset$ if

$$
L \mu+2 \pi^{2} R^{2} L^{-1} \operatorname{deg}^{2} g \leq \sqrt{2 M_{1}} \epsilon^{1-p / 2} .
$$

This gives a restriction on a degree of a monomial $g$ :

$$
R^{2}(\operatorname{deg} g)^{2} \leq \frac{L}{2 \pi^{2}} \epsilon^{1-p / 2} \sqrt{2 M_{1}}-\frac{L^{2}}{2 \pi^{2}} \mu .
$$

Note that the number $N_{n}$ of different monomials of a degree $n$ is estimated by

$$
N_{n} \geq 4 \cdot 3^{n-1} \text {. }
$$

Therefore, the homotopy complexity $\sigma=\ln \sum_{0}^{n} N_{n} \geq \ln \left(2 \cdot 3^{n}-1\right) \geq n \ln 3$. Hence, we have obtained the estimate from below for the homotopy complexity of the attractor of the system $(1.1),(1.2)$ with the potential $F_{\epsilon}$ satisfying (3.1), (3.2):

$$
\sigma^{2} \geq \frac{L \ln ^{2} 3}{R^{2} 2 \pi^{2}}\left[\sqrt{2 M_{1}} \epsilon^{1-p / 2}-\mu L\right] .
$$


For a given small $\epsilon$ the maximum of the right-hand side is at $L=\frac{\sqrt{2 M_{1}}}{2 \mu} \epsilon^{1-p / 2}$, we obtain

$$
\sigma \geq \frac{\ln 3}{2 \pi} \epsilon^{1-p / 2} \frac{M_{1}^{1 / 2}}{\sqrt{\mu} R}
$$

Obviously, if $\epsilon \rightarrow 0$ the complexity $\sigma$ tends to $+\infty$ since $p>2$.

Note that we can replace (3.5) by a condition $F_{\epsilon}(u) \geq M$ when $\operatorname{dist}(u, P) \leq$ $M^{-1 / p}$ and (3.8) takes the form

$$
\beta \leq \sqrt{2} M^{-(1-p / 2) / p}=\sqrt{2} M^{1 / 2-1 / p} .
$$

Example 3.2. We can consider in exactly the same way the case when instead of explicit formulae (3.1), (3.2) we have more general potential $F_{\epsilon}$ which satisfies conditions

$$
F_{\epsilon}(u) \geq M_{2} \epsilon^{-2} \quad \text { when }\left|u-z_{j}\right| \leq \epsilon
$$

for a fixed $\epsilon, 0<\epsilon \leq 2$. We also impose a condition

$$
F_{\epsilon}(u) \leq \mu \quad \text { when }\left|u-z_{j}\right|=R
$$

which replaces (3.9a). Repeating with obvious modifications computations of Example 3.1 (we can put $M_{2}=M_{1} \epsilon^{-p+2}$ ), we can conclude analogously to (3.11) that there exists $\beta$ such that $\mathcal{N}(g, \beta) \neq \emptyset$ if

$$
\operatorname{deg}^{2} g \leq \frac{L}{2 \pi^{2} R^{2}} \sqrt{2 M_{2}}-\frac{L^{2}}{2 \pi^{2} R^{2}} \mu
$$

and the complexity $\sigma$ is estimated as follows:

$$
\sigma^{2} \geq \frac{L \ln ^{2} 3}{2 \pi^{2} R^{2}}\left[\sqrt{2 M_{2}}-\mu L\right] .
$$

If $\epsilon$ and $M_{2}$ are fixed and the dependence of $\mu$ on $R$ is such that $\mu \leq \mu_{0} R^{-p}$, then choosing $L=\sqrt{2 M_{2}} /(2 \mu)$ we obtain $\sigma^{2} \geq \frac{\ln ^{2} 3}{4 \pi^{2} \mu_{0}} R^{p-2} M_{2}$, and complexity is large for large $R$.

Remark 3.1. In Example 3.1 we have solutions with large deg $g$ thanks to large values of $F$ as $r=\left|u-P_{i}\right| \rightarrow 0$. In Example 3.2 the same effect is thanks to small values of $F(u)$ when $|u|$ is large, in both cases the critical exponent is $|u|^{-2}$.

Example 3.3. Singular potential.

Now we shall discuss the case $\epsilon=0$. Note, that $S_{t} E_{\beta} \subset E_{\beta} \forall t \geq 0$, therefore by $(2.5)$

$$
\left|u(x)-P_{j}\right| \geq \delta \quad \forall P_{j} \in P, \quad x \in[0, L] .
$$

Therefore, if $\epsilon<\delta$, we have $u(t)$ is at the same time a solution of (1.1), (1.2) with $F=F_{\epsilon}$ and $F=F_{0}$ where

$$
F_{0}(u)=\left|u-P_{1}\right|^{-p}+\left|u-P_{2}\right|^{-p} .
$$

Let

$$
X=\left\{u_{0} \in M_{1} \text { such that } u_{0}(x) \notin P \forall x \in[0, L]\right\} .
$$

From the above discussion we conclude that for any $u_{0} \in X$ there exists $\beta>0$ such that $u_{0} \in E_{\beta}$ and $u(t) \in E_{\beta} \cap X \forall t \geq 0$. From the definition of $X$ we see, that the homotopy class $g\left(u_{0}\right)=g \in G, G=\pi_{1}\left(\mathbb{R}^{2} \backslash\left\{P_{1}, P_{2}\right\}\right)$, is well-defined. Since $u(t, x)$ is a continuous function of $(t, x) \in \mathbb{R}_{+} \times[0, L], u(t) \in g\left(u_{0}\right) \forall t \geq 0$, the 
attractor $\mathcal{A}(g, \beta)$ is not empty and contains a stable equilibrium set $\mathcal{N}(g, \beta)$ which is not empty for large $\beta$ (see Example 3.1), and is independent of $\beta$.

Remark 3.2. Existence of steady-state solutions of $\partial_{x}^{2} u=F^{\prime}(u)$ corresponding to arbitrary homotopy class $g \in \pi_{1}\left(\mathbb{R}^{1}-\left\{P_{1}, P_{2}\right\}^{*}\right)$ and to $\pi_{1}\left(\mathbb{R}^{d}-P\right)$ for $d \geq 2$ for the case of strong forces (that is of singularities of kind of (3.14)) was proved in [G].

Now we give a generalization of a preceding example. Consider a potential $F_{\epsilon}$, $0<\epsilon<r_{0}$ which is defined by the formula

$$
F_{\epsilon}(u) \geq r^{-p_{j}} \text { when }\left|u-z_{j}\right| \leq r, \quad 0<\epsilon_{0} \leq \epsilon \leq r_{0},
$$

$z_{j} \in P=\left\{z_{1}, \ldots, z_{m}\right\}$. It is assumed that $F \in C^{2}\left(\mathbb{R}^{2}\right)$.

Theorem 3.1. There exists a constant $C_{0}$ such that (3.7) holds if

$$
\beta \leq C_{0} \epsilon^{(1-p / 2)}, \quad p=\min \left(p_{1}, \ldots, p_{m}\right) .
$$

If

$$
\operatorname{deg} g \leq C_{2} L \epsilon^{1-p / 2}-C_{3} L^{2}
$$

then $Y(g, \beta) \neq \emptyset, \mathcal{A}(g, \beta), \mathcal{N}(g, \beta) \neq \emptyset$. Here $C_{2}, C_{3}$ do not depend on $\epsilon$.

The proof is analogous to considerations of Example 3.1.

The case of a singular potential (Example 3.3) can be generalized as follows. Let $F_{0} \in C^{2}\left(\mathbb{R}^{2} \backslash P\right), P=\left\{P_{1}, \ldots, P_{m}\right\}$. Let

$$
\lim _{u \rightarrow P_{1}} F(u)\left|u-P_{i}\right|^{2}=+\infty, \quad i=1, \ldots, m
$$

Let

$$
X=\left\{u_{0} \in H_{1}: u_{0}(x) \notin P \forall x \in[0, L]\right\} .
$$

Theorem 3.2. Let $u_{0} \in X$. Then there exists $\beta>0$ and a solution $u(x, t)$ of (1.1), (1.2) which belongs to $E(\beta) \cap X \forall t>0$. For every $g \in G=\pi_{1}\left(\mathbb{R}^{2} \backslash P\right)$ there exists $\beta$ such that $Y(g, \beta), \mathcal{A}(g, \beta), \mathcal{N}(g, \beta)$ are not empty.

The proof of Theorem 3.2 is quite similar to considerations of Example 3.3.

Remark 3.3. Note that the existence of steady-state solutions of (0.1) in the limit case of a singular potential when $|F(u)| \geq \mathcal{C}, F(u) /\left|u-P_{i}\right|^{2} \rightarrow \infty$ as $u \rightarrow P_{i}$ was studied in a number of papers (see [G], [ACZ], [BR] and papers cited there). Our results (restricted to steady states) differ in the following respect. We prove for this special case not only existence of steady-state solutions representing every homotopy class $g \in \pi_{1}\left\{\mathbb{R}^{d} \backslash P\right\}$, but estimate the number of trajectories which belong to a class with bounded energy $\mathcal{P}$. These estimates, as one can see, include not only characteristics of $F$ near $P$, but also far from $P$ (at the small energy level $\mu)$.

In the next example $r, R$ and $M$ are fixed, and $\mu=0$. This gives a possibility to obtain large $\operatorname{deg} g$ if $L$ is large.

Example 3.4. Let

$$
F(u)=q\left(R_{1}-\left|u-P_{1}\right|_{\epsilon}\right)^{2}\left(\left|u-P_{2}\right|_{\epsilon}-R_{2}\right)^{2}
$$

where $|\zeta|_{\epsilon}$ is a smoothened $|\zeta|,|\zeta|_{\epsilon}=|\zeta|$ if $|\zeta| \geq \epsilon, \epsilon \geq|\zeta|_{\epsilon}>\epsilon / 2$ for $|\zeta| \leq \epsilon$, $|\zeta|_{\epsilon} \in \mathcal{C}^{2}(\mathbb{R})$. In this case $\mu=0$,

$$
\mathcal{D}_{\mu}=\left\{\left|u-P_{1}\right|=R_{1}\right\} \cup\left\{\left|u-P_{2}\right|=R_{2}\right\} .
$$


We assume that $\left|P_{1}-P_{2}\right|=R_{1}+R_{2}$. Obviously, $\pi_{1}\left(\mathcal{D}_{\mu}\right)$ is the same free group with generators $g_{1}$ and $g_{2}$ as in Example 2.1.

Theorem 3.3. Let $R_{1}=R_{2}=R, r=R / 2$, let $F(u)$ be defined by (3.18) where $\epsilon$ is small enough. Let $g \in \pi_{1}\left(P^{\prime}\right)\left(P^{\prime}-\mathbb{R}^{2} \backslash P\right)$, deg $g \geq 1$. Let

$$
\operatorname{deg} g<\sqrt{\frac{L}{\sqrt{a}}} \sqrt[4]{\frac{q R^{2}}{2}} .
$$

Then $Y(\beta, g) \neq \emptyset, \mathcal{A}(\beta, g) \neq \emptyset$.

Proof. According to (3.10), $\mu=0$. One can easily see that $\mathcal{D}_{\mu}=S^{1} \vee S^{1}$ is a deformation retract of $P^{\prime}$. Since

$$
\left(\left|u-P_{2}\right|_{\epsilon}-R\right)^{2} \geq(3 R / 2-R)^{2}
$$

when $\left|u-P_{1}\right| \leq r=R / 2,\left|P_{1}-P_{2}\right|=2 R$, we can take $M=q R^{4} / 16$ in (2.6), $\beta=r \sqrt{8 a M}$. Obviously $F(u) \leq q R^{4} / 16$ when $\operatorname{dist}(u, P) \leq r$. Therefore, (2.21) takes the form

$$
2 \pi \operatorname{deg} g \leq\left(\frac{2 L}{\sqrt{a}}\right)^{1 / 2} \sqrt[4]{q R^{2} / 8}
$$

Let

$$
\lambda=\sqrt{\frac{L}{\sqrt{a}}}, \quad q^{\prime}=\sqrt[4]{q R^{2}} .
$$

We have the restriction on $g$

$$
2 \pi \operatorname{deg} g<\frac{\lambda q^{\prime}}{\sqrt[4]{2}}
$$

Remark 3.4. Obviously if $q, R, r, g$ are fixed, we can find solution $u$ of homotopy type $g$ taking $L / \sqrt{a}$ sufficiently large. Therefore (1.1) with $F$ defined by (3.10) has solutions which represent any element $g \in \pi_{1}\left(P^{\prime}, *\right)$. The same is true for

$$
F(u)=q\left(R^{2}-\left|u-P_{1}\right|^{2}\right)^{2}\left(R^{2}-\left|u-P_{2}\right|^{2}\right) .
$$

Remark 3.5. One can see considering (2.6) and (2.18) like we did in the above examples that the high complexity of attractors (which corresponds to high values of $\operatorname{deg} g$ ) may be obtained in following situations:

(a) we have large values of $M r^{2}$ (situation close to a singular);

(b) we have small values of $\mu R^{2}$, this can be achieved if $\left|P_{1}-P_{2}\right|$ is large, and the potential $F$ rapidly decays far from $P_{1}$ and $P_{2}$ (more rapidly than $\left|u-P_{i}\right|^{-2}$ );

(c) we have zero (or very close to zero) $\mu$ on the homotopy nontrivial set, surrounding $P_{1}, P_{2}$. In this case $L / \sqrt{a}$ should be large. (If $L$ is fixed, the situation is close to a singular limit $\partial_{t} u=-F^{\prime}(u)$.) 


\section{Higher Order Systems}

We consider here the case when $d \geq 2$, that is $u=\left(u_{1}, \ldots, u_{d}\right)$ takes its values in $\mathbb{R}^{d}, d \geq 2$. Results of Section 1 hold in this case. Formulations and results of Section 2 should be slightly modified.

We shall use the following notations:

$$
\mathcal{D}_{\mu}=\left\{y \in \mathbb{R}^{d}: F(y)<\mu\right\}, \quad \mathcal{D}_{\mu}^{\prime}=\mathbb{R}^{d} \backslash \mathcal{D}_{\mu} .
$$

We assume that there exists $M>\mu>0$, a local compact set $P \subset \mathcal{D}_{M}^{\prime}$ such that sets $P^{\prime}=\mathbb{R}^{d} \backslash P$ and $\mathcal{D}_{\mu}$ are homotopy equivalent, $\mathcal{D}_{\mu}$ is a deformation retract of $P^{\prime}$,

$$
\left\{u \in \mathbb{R}^{m}: \operatorname{dist}(u, P) \leq r\right\}=\Omega(P, r) \subset \mathcal{D}_{M}^{\prime} .
$$

One can easily see that these definitions agree with definitions given in Section 2.

We assume that the set $\Omega^{\prime}(P, r)=\mathbb{R}^{d} \backslash \Omega(P, r)$ and $\mathcal{D}_{\mu}$ is a deformation retract of $P^{\prime}$. In this case for $b=* \in \mathcal{D}_{\mu} \subset \Omega^{\prime}(P, r) \subset P^{\prime}$ we have

$$
\pi_{1}\left(\mathcal{D}_{\mu}, *\right)=\pi_{1}\left(\Omega^{\prime}(P, r), *\right)=\pi_{1}\left(P^{\prime}, *\right)=G .
$$

For $g \in G$ we put

$$
\begin{aligned}
X\left(\Omega^{\prime}(P, r)\right) & =\left\{u \in H_{1}([0, \ell]): u(0)=u(L)=b, u(x) \in \Omega^{\prime}(P, r) \forall x \in[0, L]\right\}, \\
X\left(\Omega^{\prime}(P, r), g\right) & =X\left(\Omega^{\prime}(P, r)\right) \cap g, \quad Y(P, r, g, \beta)=E_{\beta} \cap X\left(\Omega^{\prime}(P, r), g\right) .
\end{aligned}
$$

Here $E_{\beta}$ is defined by (1.9). We assume that conditions imposed in Section 1 on $F$ hold. For $\mathcal{D}_{\mu}^{\prime}$ given, let $r_{0}(\mu)$ be the supremum of all $r$ such that $\exists$ such $P$ that $\mathcal{D}_{\mu}^{\prime}$ is a deformation retract of $\Omega(P, r) \subset \mathcal{D}_{\mu}^{\prime}$.

Theorem 4.1. Let $R=\operatorname{dist}(b, P)$. Let $u \in H_{1}([0, L]), u(0)=b$, and

$$
\mathcal{P}(u) \leq \beta<\sqrt{2}(r-\delta) \sqrt{M}+\frac{(R-r)^{2}}{2 L} .
$$

Then

$$
\operatorname{dist}(u(x), P) \geq \delta>0 \quad \forall x \in[0, L] .
$$

Proof. To prove (4.2), assume the contrary. Then there exists $u_{0} \in E_{\beta}, x^{*}>0$ such that

$$
\operatorname{dist}\left(u\left(x^{*}\right), P\right) \leq \delta
$$

Since $u(x)$ is continuous in $x$ we can take $x^{*}$ as first moment when (4.3) holds, therefore

$$
\operatorname{dist}(u(x), P)>\delta \quad \forall x \in\left[0, x^{*}[.\right.
$$

By (4.3) and local compactness of $P$ there exists a point $P_{0} \in P$ such that

$$
\left|u\left(x^{*}\right)-P_{0}\right|=\delta
$$

Let $v(x)=u\left(x-x^{*}\right)-P_{0}$. Consider the function $\partial_{x}|v(x)|$. Since $v(x) \neq 0$ when $x<x^{*}$, this function is smooth for $0 \leq x<x^{*}$ (we use smoothness of solutions of 
(1.1) for $t>0)$. We have

$$
\begin{aligned}
\partial_{x}|v| & =\partial_{x}\left[\sum_{j=1}^{m}\left|v_{i}\right|^{2}\right]^{1 / 2} \\
& =|v|^{-1} \sum_{i=1}^{m} v_{i} \partial_{x} v_{i} \leq|v|^{-1}\left(\sum_{i=1}^{m} v_{i}^{2}\right)^{1 / 2}\left(\sum_{i=1}^{m}\left|\partial_{x} v_{i}\right|^{2}\right)^{1 / 2} \\
& =\left|\partial_{x} v\right| .
\end{aligned}
$$

Therefore, we obtain

$$
\begin{aligned}
\mathcal{P}(u) & =\int_{0}^{L}\left[\frac{a}{2}\left|\partial_{x} u\right|^{2}+F(u)\right] d x \geq \int_{0}^{x^{*}}\left[\frac{a}{2}\left|\partial_{x} v\right|^{2}+F\left(P_{0}+v\right)\right] d x \\
& \geq \int_{0}^{x^{*}}\left[\left.\frac{a}{2}\left|\partial_{x}\right| v\right|^{2}+F\left(P_{0}+v\right)\right] d x \geq \int_{0}^{x^{*}}\left[\frac{a}{2}\left|\partial_{x}\right| v||^{2}+U^{*}(P)\right] d x,
\end{aligned}
$$

where $U^{*}(P)$ is defined by (2.12). (We are using that $|v| \leq r$ implies $P+v \in$ $\left.\Omega(P, r) \subset \mathcal{D}_{M}.\right)$ Using Lemma 2.1 as in the proof of Theorem 2.1, we obtain that (4.6) and (4.2) imply $\mathcal{P}(u)>\beta$, which contradicts $u \in E_{\beta}$. This contradiction proves the theorem.

Let $b_{1}, b_{2}$ be two points in $P^{\prime}$. Let $S_{0}$ be a fixed curve in $P^{\prime}$ which connects $b_{2}$ and $b_{1}$. Obviously, any continuous curve $u(x), x \in[0, L]$ which satisfies $u(0)=b_{1}$, $u(L)=b_{2}$ determines together with $S_{0}$ a cycle in $P^{\prime}$. (We take $0=* \in[0, L]$ as a base point.) Therefore we have put in correspondence with $u(x)$ a unique element $g(u) \in \pi_{1}\left(P^{\prime}, *\right), *=b_{1} \subset P^{\prime}$ (this element depends on the choice of $\left.S_{0}\right)$.

Corollary 4.1. Let

$$
E_{\beta}=\left\{u \in M_{1}([0, L]): u(0)=b_{1}, u(L)=b_{2}\right\} .
$$

Let $S_{t}$ be the semigroup in $E_{\beta}$ defined in Theorem 1.2. Let $\beta$ satisfy (4.1) where $R=\operatorname{dist}\left\{\left\{b_{1} \cup b_{2}\right\}, P\right\}$. Then $g\left(S_{t}(u)\right)$ is well-defined for all $u \in E_{\beta}, t \geq 0$ and is constant in $t$. Every set $Y(\beta, g), g \in \pi_{1}\left(P^{\prime}, *\right)$ is invariant. If this set is non-empty, it contains the attractor $\mathcal{A}(\beta, g)$ and a stable component of equilibria $\mathcal{N}(\beta, g) \subset \mathcal{A}(\beta, g)$.

Corollary 4.2. Let $\beta$ satisfy conditions of Corollary 4.1. Then $S_{t} u_{0}$ is homotopy stable in $u_{0} \in E_{\beta}$. Namely, there exists such $\epsilon>0$ that if $u_{\theta}, \theta \in[0,1]$ is a continuous (in $\left.H_{1}([0, L])\right)$ curve connecting $u_{0}$ and $u_{1}, u_{\theta} \in E_{\beta+\epsilon} \forall \theta \in[0,1]$, then $g\left(S_{t} u_{1}\right)=g\left(S_{t} u_{0}\right) \forall t \geq 0$.

Proof. Condition (4.1) holds if $\beta$ is replaced by $\beta+\epsilon, \delta$ by $\delta / 2$. Since $S_{t} u$ continuously depends on $u$ for any fixed $t$ (see [BV]), we have a homotopy $S_{t} u_{\theta}, \theta \in[0,1]$, between $S_{t} u_{0}$ and $S_{t} u_{1}$. By Theorem $4.1 S_{t} u_{\theta}(x) \in P^{\prime} \forall x \in[0, L]$, which implies that homotopy classes of $S_{t} u_{1}$ and $S_{t} u_{0}$ coincide.

Consider now the case of periodic boundary conditions (1.2b).

Theorem 4.2. Let

$$
\beta<r \sqrt{M} \sqrt{a} / \sqrt{2}
$$


Then for every nontrivial $g \in \pi_{1}\left(\Omega^{\prime}(P, r)\right)$ (4.2) holds true with some $\delta>0$. (In the definition of $X\left(\Omega^{\prime}(P, r)^{\prime}\right)$ we impose periodic conditions (1.2b) on u instead of (1.2a).)

Proof. The proof is analogous to the case of Dirichlet boundary conditions (1.2a), therefore we show only the modifications needed.

We consider the case of periodic boundary conditions (1.2b). In this case we take $G=\pi_{1}\left(P^{\prime}\right)$ the fundamental group without base point. In the case when $g$ includes powers of different generators, we can apply the same proof to obtain (4.2). The only modification is that we take $R=\frac{1}{2} \min _{i \neq j} \operatorname{dist}\left(\Omega_{i}, r_{j}\right)$ where $\Omega_{i}$ are connected components of $\Omega(P, r)$.

The case when $g$ includes only one generator $g_{1}, g=g_{1}^{k_{1}}$ is a little different. In this case we have (4.3)-(4.5) as well. Let $X=\bigcup_{x \in[0, L]} U(x)$. Since $X \subset \Omega^{\prime}(P, r / 2)$, the estimate diam $X<r / 2$ implies $u$ is contractible in $\Omega^{\prime}(P)$. Hence, it suffices to prove the theorem for diam $X \geq r / 2$. If (4.5) holds, we have $\left|u\left(x^{*}\right)-u\left(x_{1}\right)\right| \geq r / 4$. Repeating the proof of Theorem 2.1 with $R=r$, replaced by $r / 2, r_{1}=r / 4-\delta$, we by Lemma 2.1 come to $\mathcal{P}(u) \geq \sqrt{8 a M}(r / 4-\delta)$ which gives a contradiction with (4.7) as well if $\delta$ is small. Therefore we have proved the assertion of the theorem for any nontrivial $g$.

Remark 4.1. We have the inequality

$$
\sup _{y, x \in[0, L]}|u(x)-u(y)| \leq L^{1 / 2}\left[\int_{0}^{L}\left|\partial_{x} u\right|^{2} d x\right]^{1 / 2} .
$$

Therefore, boundedness of $\mathcal{P}(u)$ implies in case of the Dirichlet boundary condition the restriction

$$
|u(x)-b| \leq \frac{\sqrt{2} L^{1 / 2}}{a^{1 / 2}} \beta \quad \forall u \in E_{\beta} .
$$

Therefore, we can replace in the formulation of Theorem 4.1 groups $\pi_{1}\left(\mathbb{R}^{d} \backslash P, *\right)$ by $\pi_{1}\left(B_{\beta} \backslash P, *\right)$ where

$$
B_{\beta}=\left\{z \in \mathbb{R}^{d}:|z-b| \leq \frac{\sqrt{2} L^{1 / 2}}{a^{1 / 2}} \beta\right\} .
$$

When periodic boundary conditions (1.2b) are imposed, (4.7) also may imply restrictions, but their form depends on structure of $P$.

Note that Definitions 2.1 and 2.2 are good for $d>2$ as well.

Theorem 4.3. The assertion of Theorem 2.2 holds for $d \geq 2$.

Note, that Remarks 2.4 and 2.5 are valid in case $d>2$.

Theorem 4.4. Assertions of Theorem 2.5 hold if we replace $\mathbb{R}^{2}$ by $\mathbb{R}^{d}$, and $\Omega(P, r)$ is defined by (4.0).

\section{Localization of Support of Homotopy Elements}

For every nontrivial element $g$ of a fundamental homotopy group $\pi_{1}\left(P^{\prime}, *\right), P^{\prime} \subset$ $\mathbb{R}^{2} \backslash\left\{P_{1} \cup P_{2}\right\}$ where $P_{1}$ and $P_{2}$ are points (see Section 3 ) we have a representation

$$
g=\prod_{j=1}^{n} g_{i_{j}}^{k_{j}}
$$


where $i_{j}=1$ or $i_{j}=2, k_{j} \in \mathbb{Z} \backslash 0$. At the same time, if $u \in g$, it is a function of $x \in[0, L]$. We will give here an algorithm which describes a location of every $N=\left|k_{1}\right|+\cdots+\left|k_{n}\right|$ of elements of decomposition of $g$ for every $u \in g$. The support of $g_{i}$ is a segment $\left[y_{i}^{-}, y_{i}^{+}\right]$, this segment is determined uniquely by $u$. Two neighboring segments $\left[y_{i}^{-}, y_{i}^{+}\right]$may overlap, but the distance between $\left[y_{i}^{-}, y_{i}^{+}\right]$and $\left[y_{i+2}^{-}, y_{i+2}^{+}\right]$is positive.

We will consider separately cases when $u$ satisfies Dirichlet and periodic boundary conditions. The case of periodic condition is more complicated, therefore we consider it in more detail. We consider $L$-periodic functions $u(x)$ which are elements of $\pi_{1}\left(P^{\prime}\right)$. Obviously, the translated function $u(x+y)$ is also periodic. At the same time, the decomposition (2.3) is not unique when we do not fix base points. We impose the condition that if $i_{1}=i_{n}$, then $k_{1}$ and $k_{n}$ have the same sign. It is possible to make cyclic permutations in this class not changing the homotopy class. We shall show connection between translations and permutations.

Now we proceed to define support of elements of $g(u)$. We use notations of Section 3. Let $Q_{1}=\left\{\left|u_{1}\right|<4 R,\left|u_{2}\right|<2 R\right\}$ be a rectangle which contains circles $\left|u-P_{i}\right|$ inside. There exists a homotopy $T_{1}: \mathbb{R}^{2} \times[0,1] \rightarrow \mathbb{R}^{2}, T_{1}(u, t)$ is continuous. $T_{1}(u, 0)=u, T_{1}$ is one-to-one for any fixed $t$ and $T_{1}\left(\mathbb{R}^{2}, 1\right)=Q_{1}$. $T_{1}$ is identical on $\left\{\left|u_{1}\right| \leq 2 R,\left|u_{2}\right| \leq R\right\}=Q_{2}$. Let $\phi(\theta, t), \theta \in[0,2],[t \in 0,1]$ be a function which is defined by

$$
\begin{gathered}
\phi(\theta, t)=\theta \quad \text { when } 0 \leq \theta \leq 1, \quad 0 \leq t \leq 1, \\
\phi(\theta, t)=1+(\theta-1)(1-t) \quad \text { when } 1 \leq \theta \leq 2, \quad 0 \leq t \leq 1 .
\end{gathered}
$$

Obviously, this function is continuous. For $u \in Q_{1}, t \in[0,1]$, let a homotopy $T_{2}$ be defined by

$$
\begin{gathered}
T_{2}(u, t)=\frac{u}{|u|} \phi(|u|, t), \quad u \neq 0, \\
T_{2}(0, t)=0 .
\end{gathered}
$$

Obviously, $T_{2}$ is identical on $Q_{2}, T_{2}$ is a retraction of $Q_{1}$ on $Q_{2}$.

Note that circles $\left|u-P_{i}\right|=R$ lie in $Q_{2}\left(P_{1}=(-R, 0), P_{2}=(R, 0)\right)$, their upper halves $\left(u_{2} \geq 0\right)$ can be explicitly written as graphs of a function $u_{2}=\psi_{1}\left(u_{1}\right)$, $-2 R \leq u_{1} \leq 2 R, 0 \leq \psi_{1} \leq R$. We define for $u \in Q_{2}, u_{2}>0$,

$$
\begin{gathered}
T_{3}\left(u_{1}, u_{2}, t\right)=\left(u_{1}, u_{2}\right) \quad \text { if } u_{2} \leq \psi_{1}\left(u_{1}\right), \\
T_{3}\left(u_{1}, u_{2}, t\right)=\left(u_{1}, u_{2} t+\psi_{1}\left(u_{1}\right)(1-t)\right)
\end{gathered}
$$

if $\psi_{1}\left(u_{1}\right) \leq u_{2} \leq R$. Similarly we define $T_{3}$ for $u_{2}<0$. This is a retraction of $Q_{2}$ on the set $Q_{3}=\left\{u: \operatorname{dist}\left\{u, P_{1} \cup P_{2}\right\} \leq R\right\}$. Let $Q_{5}=\left\{u: \operatorname{dist}\left\{u, P_{1} \cup P_{2}\right\}\right\}$ be the union of two circles (with a common point 0). For $0 \leq \rho<R$, let $Q_{4}=\{u \in$ $\left.Q_{3}: \operatorname{dist}\left\{u, P_{1} \cup P_{2}\right\}>\rho\right\}, \mathcal{D}(\rho)=\left\{u \in \mathbb{R}^{2}: \operatorname{dist}\left\{u, P_{1} \cup P_{2}\right\}>\rho\right\}$. We define a homotopy $T_{4}$ on $Q_{4}(\rho), \rho \geq 0$ separately for $u_{1} \geq 0, u_{1} \leq 0$. For $u_{1} \geq 0$ let

$$
T_{4}\left(P_{2}+v, t\right)=P_{2}+[R t+(1-t)|v|] v /|v| ;
$$

for $u_{1}<0$ this homotopy is defined in a similar way replacing $P_{2}$ by $P_{1}$. Obviously, $T_{4}\left(Q_{4}, 1\right)=Q_{5}$.

Applying homotopies $T_{1}, T_{2}, T_{3}, T_{4}$ consecutively we obtain resulting retraction $T_{5}$ of $\mathcal{D}(0)$ onto $Q_{5}$. 
Consider now a curve $u(x), x \in S^{1}=[0, L], u \in C\left(S^{1}\right), u \in \mathcal{D}(0)=P^{\prime}=R^{2} \backslash P$ $\forall x \in S^{1}$. Obviously, $u \in \pi_{1}\left(P^{\prime}\right) . v(x)=T_{5} u(x)$ is a continuous curve as well; it belongs to the same class $g$ and $v(x)$ takes values in $Q_{5}$.

Now we fix $u(x)$ and let $v(x)=T_{5} u(x)$. Let $Z$ be the set of zeros of $v$,

$$
Z=\{x \in[0, L]: v(x)=0\} .
$$

It is a compact subset of $[0, L]$. For any two points $x_{1}, x_{2} \in Z$ obviously $v\left(x_{1}\right)=$ $v\left(x_{2}\right)=0$, and restrictions of $v$ on $\left[x_{1}, x_{2}\right]$ belongs to one of the classes of $\pi_{1}\left(Q_{5}, *\right)$. We denote this class (for $v$ fixed) by $g\left[x_{1}, x_{2}\right]$. Consider pairs $x_{1}, x_{2} \in Z$ such that $0 \leq x_{1}<x_{2} \leq L$. We say that a segment $\left[x_{1}, x_{2}\right]$ is contractible if $v$ on this segment belongs to a trivial homotopy class (we denote it by 1 ), $g\left[x_{1}, x_{2}\right]=1 \in \pi_{1}\left(Q_{5}, *\right.$ ) where $*=0$ is the base point. Obviously, if $\left[x_{1}, x_{2}\right]$ and $\left[x_{2}, x_{3}\right]$ are two segments with a common point $x_{2}$, we have

$$
g\left[x_{1}, x_{2}\right] g\left[x_{2}, x_{3}\right]=g\left[x_{1}, x_{3}\right] .
$$

Hence, if both $g\left[x_{1}, x_{2}\right]$ and $g\left[x_{2}, x_{3}\right]$ are trivial, so does $g\left[x_{1}, x_{3}\right]$.

Since $v(x)$ is uniformly continuous, there exists such $\delta_{0}>0$ that if $\left|x_{1}-x_{2}\right|<\delta_{0}$, $x_{1}, x_{2} \in Z$, then $g\left[x_{1}, x_{2}\right]=1$. Our goal is to write the element $g(v)=g \in \pi_{1}\left(P^{\prime}\right)$ in the form

$$
\prod_{j=1}^{\operatorname{deg} g} g_{i_{j}}^{\delta_{1}}, \quad \delta_{j}= \pm 1,
$$

where all terms are uniquely determined by $v=T_{5} u$ and $g_{i j}=g\left[y_{j}^{-}, y_{j}^{+}\right]$. We shall define also segments $\left[y_{j}^{-}, y_{j}^{+}\right]$which correspond to elements in this decomposition of $g=g(u)=g(v) \in \pi_{1}\left(P^{\prime}\right)$.

Let $x_{1}^{-}=\inf \{x \in Z\}, x_{1}^{+}=\sup \left\{x \in Z: x_{1}^{-}+L \geq x \geq x_{1}^{-}, g\left[x_{1}^{-}, x\right]=1\right\}$. We define $x_{j}^{-}, x_{j}^{+}, j \geq 2$ by induction

Obviously,

$$
\begin{aligned}
& x_{j}^{-}=\inf \left\{x \in Z, x_{1}^{+}+L \geq x>x_{j-1}^{+}\right\}, \\
& x_{j}^{+}=\sup \left\{x \in Z, x_{j-1}^{-} \leq x \leq L+x_{1}^{-}, g\left[x_{1}^{-}, x\right]=1\right\} .
\end{aligned}
$$

$$
x_{j}^{+} \geq x_{j}^{-}, \quad x_{j-1}^{+}+\delta_{0} \leq x_{j}^{-}
$$

for all $j$. Therefore, there is only a finite number $N$ of such segments $\left[x_{j}^{-}, x_{j}^{+}\right], N \leq$ $L / \delta_{0}$, the segments $\left[x_{j}^{-}, x_{j}^{+}\right]$are uniquely defined by the above algorithm. Obviously, $g\left[x_{j-1}^{+}, x_{j}^{-}\right] \neq 1$, hence $\operatorname{deg} g\left[x_{j-1}^{+}, x_{j}^{-}\right] \geq 1$. At the same time, $\left(x_{j-1}^{+}, x_{j}^{-}\right) \cap Z=\emptyset$. This follows from the definition of $x_{j}^{-}$. This easily implies that

$$
\operatorname{deg} g\left[x_{j-1}^{+}, x_{j}^{-}\right]=1 .
$$

We denote $f_{j}=g\left[x_{j-1}^{+}, x_{j}^{-}\right], j=1, \ldots, N$. We see that $\prod_{k}^{\ell} f_{j} \neq 1$ for any $\ell \leq k \leq N$ by the definition of $x_{j}^{+}$and by (5.2). Therefore $g=\prod_{j=1}^{N} f_{j}, f_{j}=g_{k_{j}}^{\delta_{j}}, \delta_{j}= \pm 1$, $j=1, \ldots, N$ where $g_{i}$ is the generator.

A cancelling of terms is possible thanks to cyclic permutations, that is, we may have

$$
f_{j}=f_{N+1-j}^{-1}, \quad j=1, \ldots, k .
$$

Let $\bar{k}$ be the maximum of such $k, \bar{k} \geq 0$ ( $\bar{k}=0$ if such $f_{i}$ do not exist). Obviously, $N-2 \bar{k}=\operatorname{deg} g$. We put $i_{j}=k_{(j+\bar{k})}$, and obtain the representation (5.3). It is 
uniquely defined by $v$. We have segments $\left[x_{j-1}^{+}, x_{j}^{-}\right], j=\bar{k}+2, \ldots, \operatorname{deg} g+\bar{k}+1$, every segment corresponds to a generator powered by 1 or -1 .

We consider segments

$$
\left[x_{\bar{k}+j-1}^{-}, x_{\bar{k}+j}^{+}\right]=\left[y_{j}^{-}, y_{j}^{+}\right]
$$

$j=2, \ldots, \operatorname{deg} g$,

$$
\left[y_{1}^{-}, y_{1}^{+}\right]=\left[x_{N-\bar{k}}^{-}, x_{\bar{k}+1}^{+}\right] .
$$

We will call $\left[y_{j}^{-}, y_{j}^{+}\right]$the support of the element $g_{k_{j}}^{\delta_{j}}$ in (2.3). Therefore, we have proved the following theorem.

Theorem 5.1. (Support of homotopy elements). There $u$ is defined the mapping

$$
\mathcal{L}: u \rightarrow\left\{\left[y_{j}^{-}, y_{j}^{+}\right], j=1, \ldots, \operatorname{deg} g(u)\right\}
$$

such that if the homotopy class of $u$ is defined by (5.3), the restriction of $u$ on $\left[y_{j}^{-}, y_{j}^{+}\right]$belongs to has the homotopy class $g_{i_{j}}^{\delta_{j}},\left[y_{i}^{-}, y_{i}^{+}\right] \cap\left[y_{i+1}^{-}, y_{j+1}^{+}\right] \neq \emptyset, j=$ $1, \ldots, \operatorname{deg} g ;\left[y_{i}^{-}, y_{i}^{+}\right] \cap\left[y_{j+2}^{-}, y_{j+2}^{+}\right]=\emptyset, j=1, \ldots, \operatorname{deg} g ; y_{j+2}^{-}-y_{j}^{+} \geq \delta>0, \delta$ is bounded uniformly on any compact set of $u \in C\left(S^{1}\right)$.

Remark 5.1. One can easily see that the segments we have defined lie on the circle $S^{1}=\mathbb{R} / L \mathbb{Z}$.

Remark 5.2. Using the mapping $\mathcal{L}$, one can define the first element $g_{i_{1}}^{\delta_{1}}$ in the decomposition (5.3) in a following way. We take $j_{0}=\min \left\{j: y_{j}^{-} \geq 0\right\}$, and let corresponding $g_{i_{j_{0}}}^{\delta_{i_{0}}}$ be the first term in (5.3). Therefore, using the described algorithm, we can for every continuous $u(x)$, which takes values in $\mathbb{R}^{2} \backslash\left\{P_{1} \cup P_{2}\right\}$, obtain a representation (5.3) which we shall denote $\bar{g}(u)$ (any cyclic transformation of $\bar{g}$ determines the same homotopy class).

Remark 5.3. Since $F(u)$ does not depend on $x$, for any equilibrium point of (1.1), (1.2b) translated function $u(x+z)$ is a solution as well. Therefore, if $u \in \mathcal{A}(g, \beta)$, then all translations of $u$ belong to $\mathcal{A}(g, \beta)$ as well. Therefore, all words $\bar{g}$ which represent $g$ are realizable as $\bar{g}(u), u \in \mathcal{A}(g, \beta)$.

Now we consider the case of Dirichlet boundary conditions. In this case we have a base point $*=0=L$, and a base point $b=0$. The construction of support of $g_{i}$ is essentially the same with simplifications.

We denote $x_{j}^{-}, x_{j}^{+}$as follows: $x_{1}^{-}=\inf \{x \in Z\}$;

$$
\begin{aligned}
& x_{j}^{-}=\inf \left\{x \in Z, x_{j-1}^{+}<x \leq L\right\}, \\
& x_{j}^{+}=\inf \left\{x \in Z, x_{j-1}^{-} \leq x \leq L, g\left[x_{1}^{-}, x\right]=1\right\} .
\end{aligned}
$$

Also, we do not cancel $f_{j}$ and $f_{N-j}$ if they are inverse. So the mapping $\mathcal{L}$ is well-defined, and the assertion of Theorem 5.1 holds in this case as well.

The first element in (5.3) is determined homotopy invariantly (unlike in the case of periodic boundary conditions).

Remark 5.4. Localization of homotopy elements is possible in case $m>2$ as well. We have to connect $b$ by paths with circles $\left|u-P_{i}\right|=r$. The set which consists of these paths and circles (a path together with a circle is a cycle around $P_{i}$ ) is now $Q_{5}$. All other considerations are quite similar, and the mapping $\mathcal{L}: u \rightarrow$ $\left\{\left[y_{j}^{-}, y_{j}^{+}\right], j=1, \ldots, \operatorname{deg} g(u)\right\}$ is well defined. (In the case of periodic boundary conditions we make cancellations as in the case $m=2$.) 
Remark 5.5. If the argument $x$ varies increasing from 0 to $L$, it passes subsequently through segments $\left[y_{j}^{-}, y_{j}^{+}\right]$. Therefore, generators $g\left[y_{j}^{-}, y_{j}^{+}\right]^{ \pm 1}$ appear subsequently. Estimates which we give depend only on $\operatorname{deg} g$, therefore after any given at $j$-th step $g_{i_{j}}=g\left[y_{j}^{-}, y_{j}^{+}\right]^{ \pm 1}$ any new element $g_{i_{j+1}}$ may appear (excluding $g_{i_{j}}^{-1}$ ). Therefore, transitions from one pattern element $g_{i_{j}}$ to the next $g_{i_{j+1}}$ are chaotic. Since we have no natural measure describing probability of such transitions, we shall describe it (as is usually done in such situations, see [A]) using topological symbolic dynamics. We will do it in the next section.

\section{Calculation of the Homotopic Complexity FOR PERIODIC BOUNDARY CONDITIONS}

We present here a piece of symbolic dynamics which allows us to describe complexity of the attractors. Start from the description of words in the fundamental group. Under assumptions of Section 2, the group $=\pi_{1}\left(P^{\prime}, *\right)$ is the fundamental group of a bunch of circles $\bigvee_{j=1}^{m} S_{j}^{1}$ with a base point and each element of this group (except of the trivial one) is a nonreducible word $g=g_{i_{1}}^{k_{1}} g_{i_{2}}^{k_{2}} \cdots g_{i_{n}}^{k_{n}}$ where $k_{j} \in \mathbb{Z} \backslash\{0\}, j=1, \ldots, n$, and $g_{i}$ is a generator of the $j$-th group $\pi_{1}\left(S_{j}^{1}, *\right)$. At that, $\operatorname{deg} g=\left|k_{1}\right|+\cdots+\left|k_{n}\right|$. To treat periodic boundary conditions, we consider homotopies without base point. Now we describe corresponding homotopy group $G$. Here we denote $G=\pi_{1}\left(P^{\prime}\right)$, elements of $G$ are classes of equivalence. Two elements $P, g_{0} \in \pi_{1}\left(P^{\prime}, *\right)$ are equivalent, if $P=g g_{0} g^{-1}, g \in \pi_{1}\left(P^{\prime}, *\right)$. Obviously $P$ and $g_{0}$ are equivalent if corresponding words are cyclic permutations one of another. Our first problem is to calculate the number of admissible words of fixed degree $N$ taking into account the following restrictions: admissible words cannot contain combinations $g_{i} g_{i}^{-1}$ and $g_{i}^{-1} g_{i}$ for each $i=1, \ldots, m$. The language of symbolic dynamics is an adequate tool for that. Indeed, let us introduce the following topological Markov chain. It has $2 m$ states denoted by symbols $1, \ldots, 2 m$. We identify symbols $1, \ldots, m$ with generators $g_{1}, \ldots, g_{m}$ and symbols $m+1, \ldots, 2 m$ with elements $g_{1}^{-1}, \ldots, g_{m}^{-1}$ correspondingly. There is a transition $i \rightarrow j, i, j \in\{1, \ldots, 2 m\}$, if the pair $(i, j)$ does not correspond to pair $g_{k} g_{k}^{-1}$ or $g_{k}^{-1} g_{k}, k \in\{1, \ldots, m\}$. Then, as usual, consider the matrix of transition, say $A=\left(a_{i j}\right)_{i, j=1}^{2 m}$, so that $a_{i j}=1$ if there exists a transition $i \rightarrow j$, and $a_{i j}=0$ if not. For example for $m=2$ this matrix has the following form

$$
A=\left(\begin{array}{llll}
1 & 1 & 0 & 1 \\
1 & 1 & 1 & 0 \\
0 & 1 & 1 & 1 \\
1 & 0 & 1 & 1
\end{array}\right)
$$

Recall that we call homotopy complexity of the set of functions the log of number of homotopy classes to which the functions belong. Then denote by $\Omega_{m}$ the set of infinite sequences $\underline{\omega}=\left(\ldots \omega_{-1} \omega_{0} \ldots \omega_{k} \ldots\right), \omega_{k} \in\{1, \ldots, 2 m\}$, with the following property $a_{\omega_{k} \omega_{k+1}}=1, k \in \mathbb{Z}$, and endow it with the metric

$$
\operatorname{dist}\left(\underline{\omega}^{\prime}, \underline{\omega}^{\prime \prime}\right)=\sum_{k=-\infty}^{\infty} \frac{1}{2^{|k|}}\left|\omega_{k}^{\prime}-\omega_{k}^{\prime \prime}\right| .
$$

After that $\Omega_{m}$ becomes a compact Cantor-like metric space and the shift map $\sigma: \Omega_{m} \rightarrow \Omega_{m}$ defined by $(\sigma \underline{\omega})_{k}=\omega_{k+1}$ if $(\underline{\omega})_{k}=\omega_{k}$, becomes a homeomorphism. 
The dynamical system $\left(\sigma^{n}, \Omega_{m}\right)_{n \in \mathbb{Z}}$ is called the topological Markov chain (or subshift of finite type) determined by the matrix of transitions $A$ (see, for instance $[\mathrm{A}]$ ). It is very simple to express the number of admissible words of length $N$, say $K_{N}$, using the matrix $A$. Let us identify an admissible word $g=g_{i_{1}}^{k_{1}} g_{i_{2}}^{k_{2}} \ldots g_{i_{n}}^{k_{n}}$, deg $g=N$ with the corresponding word $\omega_{0}^{\prime} \omega_{1}^{\prime} \cdots \omega_{N-1}^{\prime}, \omega_{i}^{\prime} \in\{1, \ldots, 2 m\}$, and, then, with the periodic sequence $\underline{\omega}=\left(\ldots \omega_{0} \ldots \omega_{N-1} \ldots \omega_{k} \ldots\right)$ where $\omega_{0}^{\prime}=\omega_{0}, \ldots, \omega_{N-1}^{\prime}=$ $\omega_{N-1} ; \omega_{N}=\omega_{0}^{\prime}, \ldots, \omega_{-1}=\omega_{N-1}^{\prime}, \ldots$ It is clear that $\sigma^{N} \underline{\omega}=\underline{\omega}$, so $\underline{\omega}$ is a $N$-periodic point of the map $\sigma$. And therefore the number $K_{n}$ of admissible words of $\operatorname{deg} N$ coincides with the number of $N$-periodic points of the topological Markov chain $\left(\sigma^{n}, \Omega_{2 m}\right)$. It is known, see for example [A], that $K_{N}=\lambda_{1}^{N}+\lambda_{2}^{N}+\cdots+\lambda_{2 m}^{N}$ where $\lambda_{1}, \lambda_{2}, \ldots, \lambda_{2 m}$ are eigenvalues of the matrix of transitions $A$. Simple calculations show us that

$$
\left(\lambda_{1}, \ldots, \lambda_{2 m}\right)=(\underbrace{1, \ldots, 1}_{m}, \underbrace{-1,-1, \ldots,-1}_{m-1}, 2 m-1)
$$

Therefore

$$
\begin{aligned}
K_{N} & =m+(m-1) \cdot(-1)^{N}+(2 m-1)^{N} \\
& = \begin{cases}(2 m-1)^{N}+1 & \text { if } N \text { is odd } \\
(2 m-1)^{N}+(2 m-1) & \text { if } N \text { is even. }\end{cases}
\end{aligned}
$$

For $m=2, K_{N}=3^{N}+2+(-1)^{N}$. The topological entropy of the shift map $\left.\sigma\right|_{\Omega_{2 m}}$ equals $\ln (2 m-1)$. It follows from $(6.1)$ that the number of all admissible words $g$ with $1 \leq \operatorname{deg} g \leq N$ is

$$
\begin{aligned}
\sum_{k=1}^{N} m & +(m-1)(-1)^{k}+(2 m-1)^{k} \\
& =N m+\frac{1}{2}\left[(m-1)\left(1+(-1)^{N}\right)+\frac{(2 m-1)}{m-1}\left((2 m-1)^{N}-1\right)\right] .
\end{aligned}
$$

We call localized complexity the $\log$ of number of words $\mathcal{L} u$ for all $u$ in a set of functions ( $\mathcal{L}$ is defined in Theorem 5.1). Taking into account Remark 5.3, we see that the localized homotopic complexity of an attractor which contains solutions of all homotopic classes up to $\operatorname{deg} g \leq N$ and their translations can be estimated as follows:

$$
\begin{aligned}
\mathcal{K}(A) & =\ln \left[N m+\frac{1}{2}\left[(m-1)\left(1+(-1)^{N}\right)+\frac{2 m-1}{m-1}\left((2 m-1)^{N}-1\right)\right]\right] \\
& \cong N \ln (2 m-1) .
\end{aligned}
$$

For $m=2$

$$
\mathcal{K}(A)=\ln \left(2 N+\frac{1}{2}\left(3^{N+1}+(-1)^{N}-2\right)\right) .
$$

Let us calculate now the number of all admissible words $g$ with $N_{1} \leq \operatorname{deg} g \leq N_{2}$. This number, say $\mathcal{K}_{N_{1} N_{2}}$, is obtained directly from the formula (6.1):

$$
\mathcal{K}_{N_{1} N_{2}}=\mathcal{K}_{N_{2}}-\mathcal{K}_{N_{1}}=(2 m-1)^{N_{2}}-(2 m-1)^{N_{1}}+(m-1)\left((-1)^{N_{2}}-(-1)^{N_{1}}\right) .
$$


For $m=1$

$$
\mathcal{K}_{N_{1} N_{2}}=3^{N_{2}}-3^{N_{1}}+2\left((-1)^{N_{2}}-(-1)^{N_{1}}\right) .
$$

The number $\ln \mathcal{K}_{N_{1} N_{2}}$ is, in fact, the homotopic complexity in the fixed basis of a piece of the attractor $\mathcal{A}$ corresponding to solutions with energy between two levels: $E_{1}$ which is related to $\operatorname{deg} g=N_{1}$ and $E_{2}$ - related to $\operatorname{deg} g=N_{2}$.

Now, consider the problem of homotopic complexity of attractors (without localization $\mathcal{L}$ of homotopy elements of a basis). Instead of words determined by elements of the fundamental group $\pi_{1}\left(P^{\prime}, *\right)$ we need to calculate the number of homotopy classes of free homotopies. It means that a word $g^{\prime}$ corresponding to the symbolic word $\left(\omega_{0}^{\prime}, \ldots, \omega_{N-1}^{\prime}\right)$ and a word $g^{\prime \prime}$ corresponding to the word $\left(\omega_{0}^{\prime \prime}, \ldots, \omega_{N-1}^{\prime \prime}\right)$ represent the same class of free homotopies iff the first of them is a result of a cyclic permutation of the second one.

At first, let us assume that $N$ is a prime number. Then each $N$-periodic trajectory of the shift map $\sigma$ contains strictly $N, N$-periodic points, and every class of free homotopies of degree $N$ corresponds to one and only one $N$-periodic trajectory. Therefore, the number of classes of free homotopies with degree $N$, say $\widetilde{\mathcal{K}}_{N}$, coincides with the number of $N$-periodic trajectories and

$$
\widetilde{\mathcal{K}}_{N}=\frac{1}{N} \mathcal{K}_{N}=\frac{1}{N}\left(m+(m-1)(-1)^{N}+(2 m-1)^{N}\right) .
$$

The homotopic complexity of a piece of an attractor $\mathcal{A}$ which contains solutions corresponding to words $g$ with $\operatorname{deg} g=N$ is the following number

$$
\begin{aligned}
\widetilde{\mathcal{K}}(\mathcal{A}) & =\ln \widetilde{\mathcal{K}}_{N}=\ln \frac{1}{N}\left(m+(m-1)(-1)^{N}+(2 m-1)^{N}\right) \\
& \cong N \ln (2 m-1)-\ln N .
\end{aligned}
$$

Assume now that $N$ is not a prime number: $N=N_{1} \cdot N_{2}, N_{1}>1, N_{2}>1$. Then an $N$-periodic point could be the $N_{1}$-periodic one. Therefore, the corresponding trajectory contains at most $N_{1}$ points. Since $N_{1}<N$ then the number of periodic trajectories in this case is greater than $\frac{1}{N} \mathcal{K}_{N}$, and we have the following estimation for the homotopic complexity of the piece $\mathcal{A}_{N}$ of an attractor corresponding to the word with $\operatorname{deg} g=N$.

$$
\begin{aligned}
& \ln \frac{1}{N}\left(m+(m-1)(-1)^{N}+(2 m-1)^{N}\right) \\
& \quad \leq \widetilde{\mathcal{K}}\left(\mathcal{A}_{N}\right) \leq \ln \left(m+(m-1)(-1)^{N}+(2 m-1)^{N}\right) .
\end{aligned}
$$

This estimation works for every $N \geq 1$, so after summation we obtain the following estimation of homotopic complexity of an attractor $\mathcal{A}$ which contains solutions of homotopic type $g, \operatorname{deg} g \leq N$ :

$$
\begin{aligned}
\ln \sum_{k=1}^{N} \frac{1}{k}\left(m+(m-1)(-1)^{k}+(2 m-1)^{k}\right) \\
\quad \leq \widetilde{\mathcal{K}}(\mathcal{A}) \leq \ln \sum_{k=1}^{N}\left(m+(m-1)(-1)^{k}+(2 m-1)^{k}\right) .
\end{aligned}
$$

In fact for any number $N$ we can find not only estimations like (6.9), (6.10) but rigorous formulas. These formulas look very cumbersome, so let us only explain how to do it. We consider 2 examples. Let $N=N_{1}^{2}$, first, and $N_{1}$ be a prime. We may separate all $N$-periodic points into two sets: the first one contains only $N$-periodic 
points each of those is $N_{1}$-periodic, the second one contains others. The numbers of trajectories (in other words the number of different classes of free homotopies) corresponding to the first class is $\frac{1}{N_{1}} \widetilde{\mathcal{K}}_{N_{1}}$ and corresponding to the second one is $\frac{1}{N}\left(\mathcal{K}_{N}-K_{N_{1}}\right)$. Using formula (6.1) we have that the corresponding complexity can be represented in the following form (compare with (6.9))

$$
\begin{aligned}
\widetilde{\mathcal{K}}\left(\mathcal{A}_{N}\right)=\ln & \left\{(2 m-1)^{N} \cdot \frac{1}{N}+(2 m-1)^{N_{1}}\left(\frac{1}{N_{1}}-\frac{1}{N}\right)\right. \\
& \left.+(m-1)\left((-1)^{N} \cdot \frac{1}{N}+(1)^{N_{1}} \cdot\left(\frac{1}{N_{1}}-\frac{1}{N}\right)\right)+\frac{1}{N_{1}} m\right\} .
\end{aligned}
$$

Consider the second example. Let $N=N_{1} \cdot N_{2}$ where $N_{1}, N_{2}$ be different primes. In the same way as above we separate all $N$-periodic points into three sets: (1) the $N_{1}$-periodic, $(2)$ the $N_{2}$-periodic, (3) the others. The number of trajectories corresponding to the first set is $\frac{1}{N_{1}} K_{N_{1}}$, the second one is $\frac{1}{N_{2}} K_{N_{2}}$ and the third one is $\frac{1}{N}\left(K_{N}-K_{N_{1}}-K_{N_{2}}\right)$. Therefore, the corresponding homotopic complexity using formulas (6.1) and (6.7) may be written in the form

$$
\begin{aligned}
\widetilde{\mathcal{K}}\left(\mathcal{A}_{N}\right)=\ln & {\left[\sum _ { i = 1 } ^ { 2 } \frac { 1 } { N _ { i } } \left(m+(m-1)(-1)^{N_{i}}+(2 m-1)^{N_{i}}\right.\right.} \\
& +\frac{1}{N}\left(m+(m-1)^{N}+(2 m-1)^{N}\right. \\
& \left.-\sum_{i=1}^{2} m+(m-1)(-1)^{N_{i}}+(2 m-1)^{N_{i}}\right] .
\end{aligned}
$$

In the same way we can derive the formula of complexity for the general case $N=N_{1}^{\alpha_{1}} \cdot N_{2}^{\alpha_{2}} \cdots N_{s}^{\alpha_{s}}$.

Remark 6.1 . One can easily see by (6.1), that for any $N$

$$
\widetilde{\mathcal{K}}\left(\mathcal{A}_{N}\right) \geq \ln \left[K_{N} / N\right]=\ln \left[m+(m-1)(-1)^{N}+(2 m-1)^{N}\right]-\ln N .
$$

For a prime $N$ this estimate is precise (see (6.7)).

\section{REFERENCES}

[A] V. M. Alekseev, Symbolic Dynamics, XI Summer School of Academy of Science of Ukraine, Kiev, Naukova Dumka (1976). MR 57:4249

[AC] V. S. Afraimovich and S.-N. Chow, Criteria of spatial chaos in lattice dynamical systems, Preprint CDSNS 93-142 (1993).

[ACZ] A. Ambrosetti, V. Coti-Zelati, Closed orbits of fixed energy for singular Hamiltonian systems, Arch. Rat. Mech. Anal. 112 (1990), 339-362. MR 91k:34053

[BBH] F. Bethuel, N. Brezis and F. Helein, Ginzburg-Landau Vortices, Birkhäuser, 1994. MR 95c:58044

[BV] A. V. Babin, M. I. Vishik, Attractors of Evolution Equations, North-Holland, 1991. MR 92j:35016

[B] A. Bahri, The variational contribution of the periodic orbits provided by Birkhoff-Lewis Theorem (with applications to convex Hamiltonians and to three-body type problems), Duke Math. J. 70 (1993), 1-205. MR 94g:58036

[FFG] A. T. Fomenko, D. G. Fuchs, V. V. Gutenmacher, Homotopic Topology, Academiai Kiado, Budapest, 1986. MR 88f:55001

[G] W. B. Gordon, Conservative dynamical systems involving strong forces, Trans. Amer. Math. Soc. 204 (1975), 113-135. MR 51:14152 
[Ha] J. K. Hale, Asymptotic behavior of dissipative systems, Math. Surveys and Monographs, vol. 25, AMS, 1988. MR 89g:58059

[Ham] R. Hamilton, Harmonic Maps of Manifolds with Boundary, Lecture Notes in Mathematics, vol. 471, Springer Verlag, 1975. MR 58:2872

[H] D. Henry, Geometric Theory of Parabolic Equations, Lecture Notes in Math., vol. 840, Springer-Verlag, 1981. MR 83j:35084

[JM] S. Jimbo and Y. Morita, Stability of nonconstant steady-state solutions to a GinzburgLandau equation in higher space dimensions, Nonlinear Anal. TMS 22 (1994), 753-770. CMP 94:10

[KW] K. Kishimoto and M. F. Weinberger, The spatial homogeneity of stable equilibria of some reaction diffusion systems on convex domain, J. Differential Equations $\mathbf{5 8}$ (1985), 15-21. MR 87a:35105

[L] J. L. Lions, Quelques méthodes de résolution des problemes aux limites non lineaires, Dunod, Paris, 1969. MR 41:4326

[M] W. S. Massey, Algebraic Topology: An Introduction, Harcourt Brace and World, Inc., New York, 1967. MR 35:2271

[N] Y. Nishiura, Coexistence of infinitely many stable solutions to reaction diffusion systems in the singular limit, Dynamics Reported New Series, No. 3 (1994), 25-103.

[R] P. M. Rabinowitz, Periodic solutions of Hamiltonian systems, Comm. Pure Appl. Math. 31 (1978), 157-184. MR 57:1674

[W] G. W. Whitehead, Elements of Homotopy Topology, Springer-Verlag, 1978. MR 80b: 55001

CDSns, Georgia Institute of Technology, Atlanta, Georgia 30332-0190

Moscow State University of Communications, Obraztsova 15, 101475 Moscow, Russia

CDSNS and School of Mathematics, Georgia Institute of Technology, Atlanta, Georgia 30332-0160 